LAWRENCE LIVERMORE N A T IO N A L LABORATORY
Model-based Processing of Micro-cantilever Sensor Arrays

J. W. Tringe, D. S. Clague, J. V. Candy, C. L. Lee, R. E. Rudd, A. K. Burnham

November 23, 2004 
This document was prepared as an account of work sponsored by an agency of the United States Government. Neither the United States Government nor the University of California nor any of their employees, makes any warranty, express or implied, or assumes any legal liability or responsibility for the accuracy, completeness, or usefulness of any information, apparatus, product, or process disclosed, or represents that its use would not infringe privately owned rights. Reference herein to any specific commercial product, process, or service by trade name, trademark, manufacturer, or otherwise, does not necessarily constitute or imply its endorsement, recommendation, or favoring by the United States Government or the University of California. The views and opinions of authors expressed herein do not necessarily state or reflect those of the United States Government or the University of California, and shall not be used for advertising or product endorsement purposes.

This work was performed under the auspices of the U.S. Department of Energy by University of California, Lawrence Livermore National Laboratory under Contract W-7405-Eng-48. 


\title{
MODEL-BASED PROCESSING OF MICRO-CANTILEVER SENSOR ARRAYS
}

\author{
J. Tringe, D. Clague, J. Candy, C. Lee, R. Rudd, and A. Burnham \\ University of California \\ Lawrence Livermore National Laboratory \\ Livermore, CA 94550 \\ Executive Summary
}

\begin{abstract}
We develop a model-based processor (MBP) for a micro-cantilever array sensor to detect target species in solution. After discussing the generalized framework for this problem, we develop the specific model used in this study. We perform a proof-of-concept experiment, fit the model parameters to the measured data and use them to develop a Gauss-Markov simulation. We then investigate two cases of interest: (1) averaged deflection data, and (2) multi-channel data. In both cases the evaluation proceeds by first performing a model-based parameter estimation to extract the model parameters, next performing a Gauss-Markov simulation, designing the optimal MBP and finally applying it to measured experimental data. The simulation is used to evaluate the performance of the MBP in the multi-channel case and compare it to a "smoother" ("averager") typically used in this application. It was shown that the MBP not only provides a significant gain $(\sim 80 \mathrm{~dB})$ in signal-to-noise ratio (SNR), but also consistently outperforms the smoother by $40-60 \mathrm{~dB}$. Finally, we apply the processor to the smoothed experimental data and demonstrate its capability for chemical detection. The MBP performs quite well, though it includes a correctable systematic bias error.
\end{abstract}

The project's primary accomplishment was the successful application of model-based processing to signals from micro-cantilever arrays: 40-60 dB improvement vs. the smoother algorithm was demonstrated. This result was achieved through the development of appropriate mathematical descriptions for the chemical and mechanical phenomena, and incorporation of these descriptions directly into the model-based signal processor. A significant challenge was the development of the framework which would maximize the usefulness of the signal processing algorithms while ensuring the accuracy of the mathematical description of the chemical-mechanical signal. Experimentally, the difficulty was to identify and characterize the non-target signals present in the measurement system. In the future, these signals will limit the ability of the sensor to detect very small quantities of chemicals generated by nuclear processing. In this project, it became necessary to make use of a model system, mercaptoethanol, which created a large, reproducible signal that could be readily analyzed with the model-based processor. Further, redundant cantilevers were examined exclusively: all levers were nominally identically functionalized, and no "control" levers were used that did not react to the mercaptoethanol signal. To demonstrate the full utility of the MBP for chemical sensing, the logical and necessary next steps are (1) verify the physical models used in this study for a variety of solvents and target molecules (this data has already been obtained as part of this study) (2) make use of control levers, and (3) extend the experimental library to include low concentrations of chemical targets of practical interest for sensing nuclear processes.

\section{Outline:}

1. Introduction

2. Experimental description

3. Physical chemistry

4. Mechanical modeling

5. Signal processing

6. Summary

7. Appendix A: Derivation of adsorption and desorption parameters 


\section{Introduction: Cantilever Sensor Arrays}

Micro-machined cantilevers can function as detection devices when one side is fabricated to be chemically distinct from the other, as shown in Fig. 1.

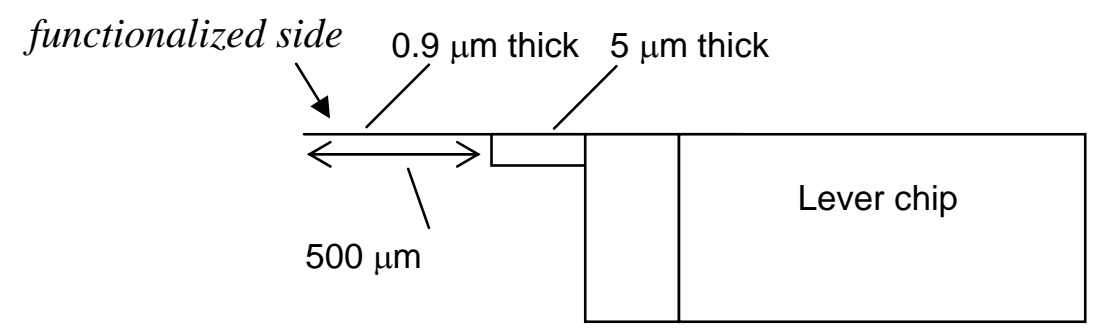

Fig. 1: Side view of typical micro-machined cantilever showing cantilever dimensions: 0.9 microns thick, 500 microns long (and 100 microns wide - not shown). To function as a sensor, the top of the cantilever ("functionalized side") must be made to be chemically distinct from the bottom.

Functionalization can be accomplished, for example, by evaporating a thin ( 10's of nm) film of metal such as Au on the top of the chip, then immersing the cantilever chip in a "probe" chemical that will bind preferentially to the Au thin film. The lever acts as a sensor when it is exposed to a second, "target" chemical that reacts with the probe, since the reaction causes a free energy change that induces a surface stress at the cantilever surface. Differential surface stress, $\Delta \sigma$, in turn, induces a deflection of the cantilever that can be measured optically or electronically

The total free energy change at the surface, $\Delta \mathrm{G}$, can described as the free energy due to four contributions: $\Delta \mathrm{G}_{\mathrm{CANT}}$, the mechanical energy associated with bending the cantilever, $\Delta \mathrm{G}_{\mathrm{POLY}}$, free energy resulting from macromolecular conformational entropy and nonelectrostatic interactions, $\Delta \mathrm{G}_{\mathrm{OSM}}$, free energy from osmotic pressure of counterions near the surface of the cantilever, $\Delta \mathrm{G}_{\mathrm{ELEC}}$, the electrostatic free energy [1].

Eq. 1

$$
\Delta \mathrm{G}=\Delta \mathrm{G}_{\mathrm{CANT}}+\Delta \mathrm{G}_{\mathrm{POLY}}+\Delta \mathrm{G}_{\mathrm{OSM}}+\Delta \mathrm{G}_{\mathrm{ELEC}}
$$

The free energy change is related to $\Delta \sigma$, the stress difference between top and bottom side of the $i^{\text {th }}$ cantilever by:

Eq. 2

$$
\Delta \sigma_{i}(t)=\Delta G_{i}(t) \Gamma_{i}(t)
$$

where $\Delta \mathrm{G}$ has units of $\mathrm{J} / \mathrm{mole}$ and is the sum of all of the contributions to free energy changes at the surface of the $i^{\text {th }}$ cantilever, $\Gamma_{\mathrm{i}}(\mathrm{t})$ is the surface concentration of the species of interest (in numbers of species per $\mathrm{cm}^{2}$ ) on the surface of the $i^{\text {th }}$ cantilever $[2,3]$. 
The surface concentration of the interacting molecules, $\Gamma_{\mathrm{i}}(\mathrm{t})$, is estimated using Langmuir kinetics, e.g., the first-order Langmuir kinetics has the following form:

Eq. 3

$$
\frac{d(\Theta)}{d t}=k_{a} c(t)(1-\Theta)-k_{d} \Gamma_{\max } \Theta
$$

Here $\Theta$ is $\frac{\Gamma(t)}{\Gamma_{\text {max }}}, \mathrm{c}(\mathrm{t})$ is the bulk concentration of the target molecule in solution in moles per liter, or [M], $k_{a}$ is the adsorption rate constant in $[\mathrm{M}]^{-1} \mathrm{~s}^{-1}, k_{d}$ is the desorption rate constant in $\mathrm{cm}^{2} \#$ species ${ }^{-1} \mathrm{~s}^{-1}$, and $\Gamma_{\max }$ is the maximum possible surface concentration of the species of interest in actual numbers of species per $\mathrm{cm}^{2}$. Please see Appendix A for a more detailed explanation of Eq. 3.

Finally, differential surface stress in the cantilever induces a deflection on the $i^{\text {th }}$ cantilever, $\Delta \mathrm{z}_{\mathrm{i}}(\mathrm{t})$, according to Stoney's equation:

Eq. 4

$$
\Delta \sigma_{i}(t)=\frac{4}{3(1-v)} \frac{l}{W \times t} k_{r e c t} \Delta z_{i}(t)
$$

where $v$ is Poisson's ratio, $l, W$ and $t$ are the rectangular cantilever length, width and thickness, respectively, $k_{\text {rect }}$ is the cantilever spring constant.

Cantilevers can function as effective sensors in liquid or gas. In a gas environment, target molecules bind to an oscillating cantilever and produce resonant frequency shifts that can be directly linked to the mass of the target. Resonant frequency shift measurements are difficult to measure in liquid due to damping effects, however. Cantilever sensors in liquid therefore generally rely on measured DC deflection signals of non-oscillating levers. This report pertains primarily to cantilever sensors operating in liquids, although some aspects of the signal processing algorithm may be applicable to cantilever arrays in gas environments as well.

A limitation on cantilever sensors in liquid is that their signal-to-noise ratio (SNR) is rather low, often 5:1 or smaller. SNR is expected to be significantly lower in many operational environments of interest. Further, the reliability of fielded cantilever sensors ultimately depends on their being incorporated into arrays, which increase system complexity and can make response interpretation difficult. However, these difficulties are exactly the type that can be overcome with effective signal extraction techniques such as the model-based approach, the subject of this invention. In this report, the modelbased signal extraction system for cantilever arrays is described that takes advantage of the fact that multiple redundant signals are available to the signal processing algorithm. 


\section{Experimental description}

In this section, the cantilever detection experimental apparatus will be described, together with results that demonstrate detection of sulfur-terminated molecules with Aucoated levers. Non-chemical signals are measured and characterized so that these can be separated from the chemical signals of interest.

\subsection{Experimental description: effects of refractive index differences}

An eight-cantilever array chip, obtained from Veeco Instruments, was used for this experiment. Actual lever deflections were not measured; the goal was to determine what variability exists in the Scentris optical detection system when fluids of differing indices of refraction are used in the flow cell. To accomplish this, apparent deflections were observed as light beams were focused on the rigid base of the chip. The Scentris system uses super-luminescent diodes (SLD) to generate non-monochromatic light, which is reflected by the cantilevers into a position-sensitive detector optimized for light with an average wavelength of about $940 \mathrm{~nm}$ (Fig. 2).

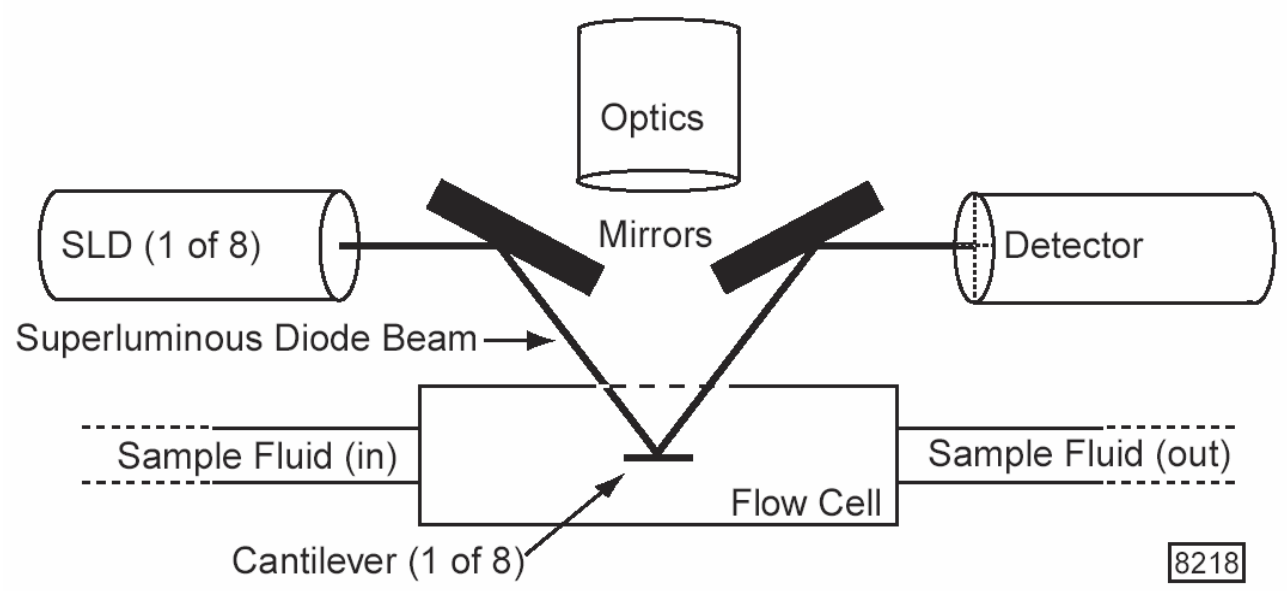

Fig. 2: Scentris optical detection system. (Diagram from Scentris Application Notes, Veeco Instruments.)

The lever chip had $1.5 \mathrm{~nm}$ Ti and $20 \mathrm{~nm}$ Au evaporated onto the surface. Immediately prior to being loaded into the flow cell, the chip was cleaned in piranha (1:3, hydrogen peroxide: sulfuric acid) for 20 minutes, then rinsed in $10 \mathrm{ml}$ water for $\sim 10 \mathrm{~min}$. The chip was then placed directly into the freshly-cleaned flow cell, which was flooded with water and sealed.

Light was focused on the chip at the base of the cantilever, as shown in Figs. 3 and 4. It appears that the Au surface may have been damaged somewhat by the extended piranha cleaning (some gold delamination appears to have occurred), but the areas used for this experiment were smooth and reflective. The actual position of the light spot on the cantilever is also visible as a white spot in Fig. 3. The positions all of the light spots used during the experiments are shown by green circles in Fig. 3, and by a green line in 
the side view of the chip shown in Fig. 4. Note from Fig. 4 that the "levers" are actually 525 microns thick under the light spots as they were positioned for these measurements. Due to the fact that there is a damaged area on the surface of the third lever from the top in Fig. 3, this lever was not used for these experiments.

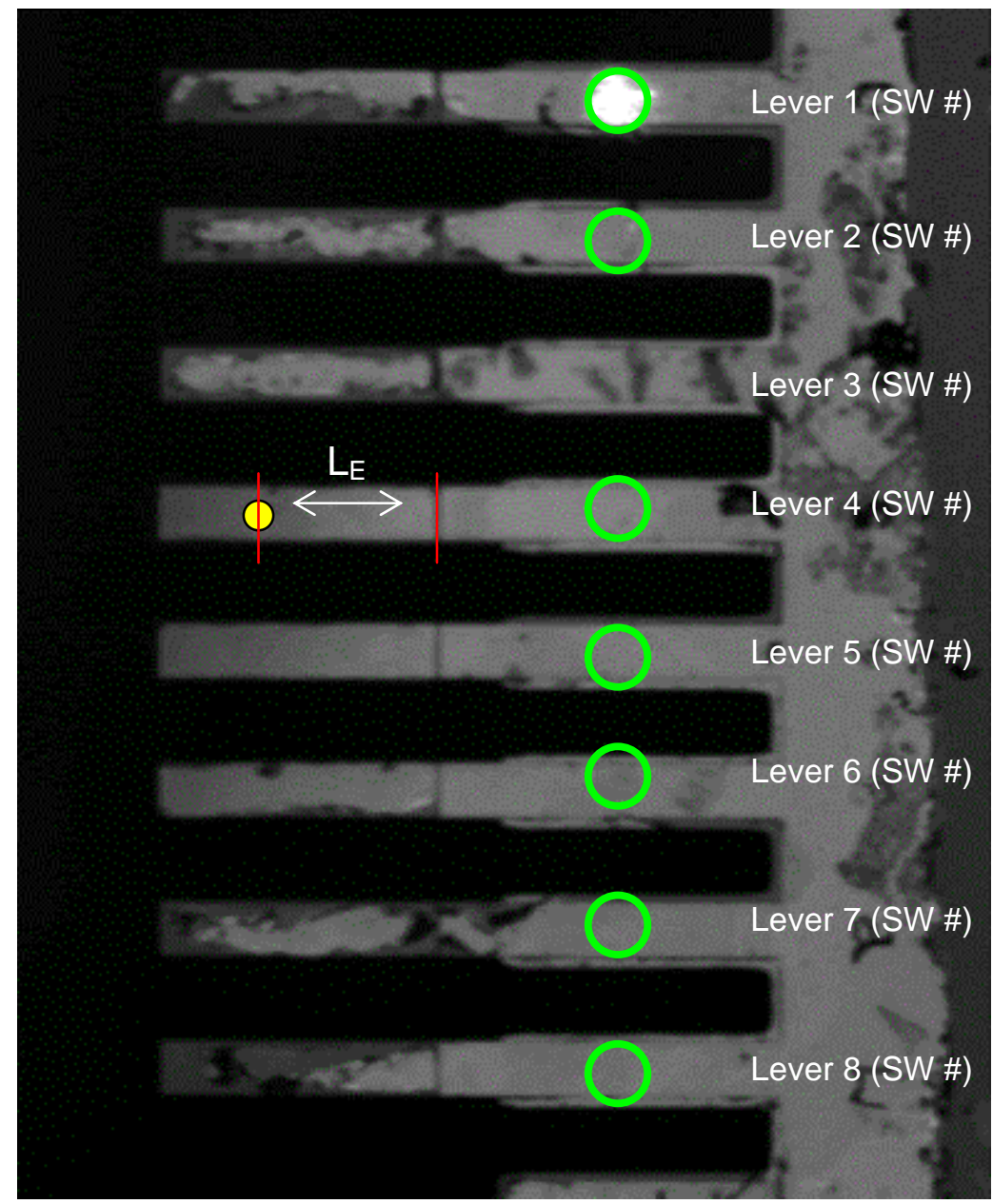

Fig. 3: Top view of light spot position and cantilevers; levers are numbered according to software (SW) scheme, reverse of etched numbers on chip.

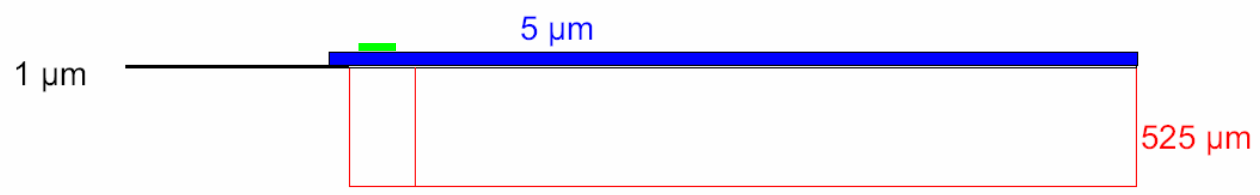

Fig. 4: Side view of lever chip showing SLD spot position in green. 
The Scentris software requires that an "effective length" for the cantilever be entered so that deflection signals may be appropriately calibrated. For typical Scentris experiments, this effective length is measured from an etched mark on the top of the chip to the center of the light spot, shown schematically in Fig. 3 with the light spot

represented by a yellow dot. (The etched mark is under the red line on the right.) For this experiment, however, the effective length is not physically meaningful since the light spot is not being positioned on the flexible part of the cantilever. However, an effective length of $460 \mathrm{~nm}$ was entered into the software so that the magnitude of the calculated "deflection" signals might be appropriately interpreted when comparing with actual lever deflection experiments, where effective lever lengths of about $460 \mathrm{~nm}$ are common.

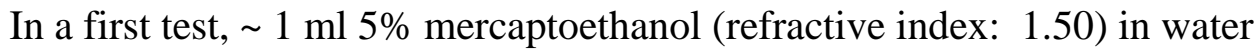
(refractive index: 1.33) was manually injected into through the 50 microliter flow cell in about 10 sec. An apparent deflection occurred, as shown in Fig. 5. The magnitude of the apparent deflection varied from less than $10 \mathrm{~nm}$ for most levers to $20 \mathrm{~nm}$ for one lever.

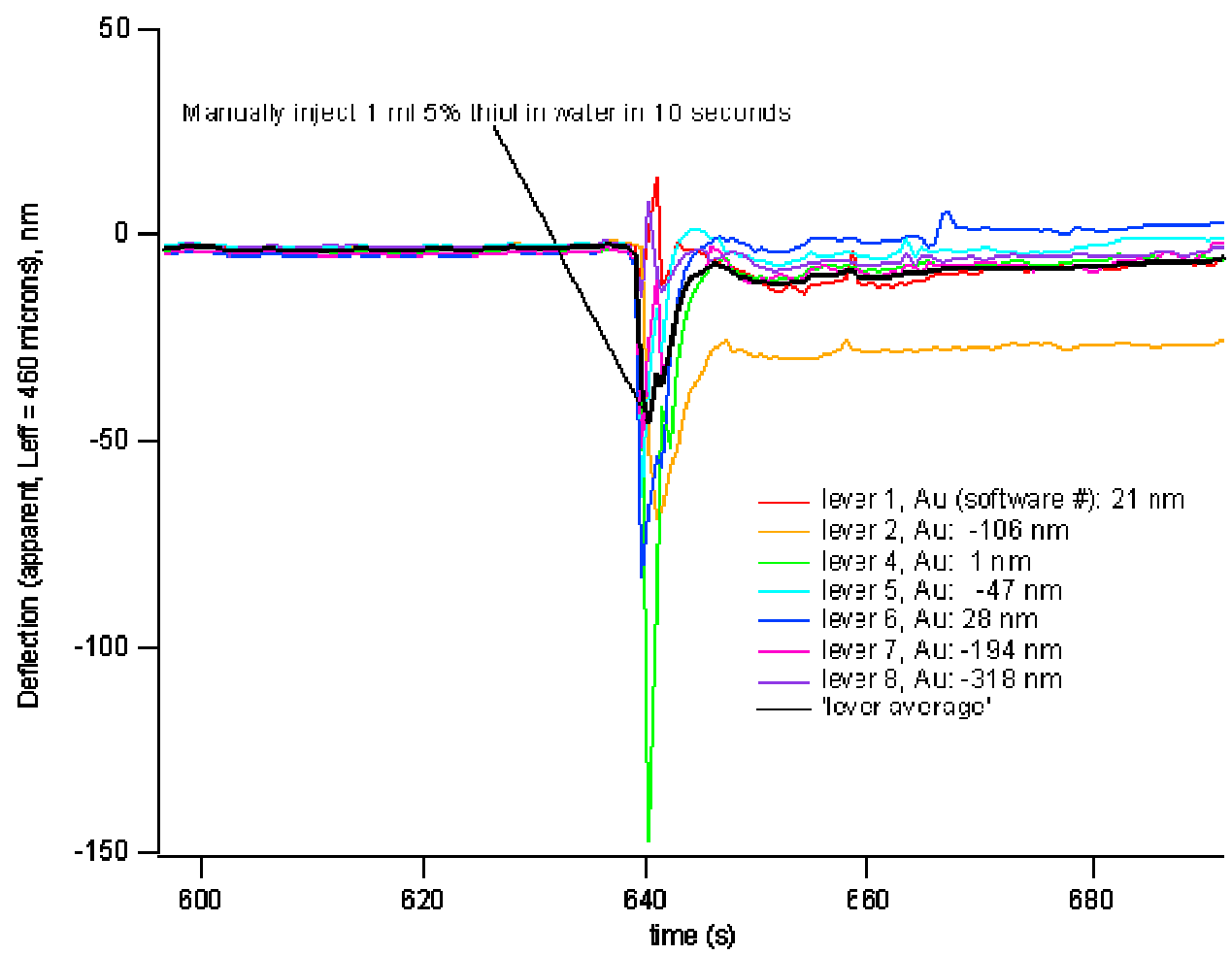

Fig. 5: Effect of injecting 5\% mercaptoethanol in water into the flow cell which previously contained pure water. Nanometer values in the legend are the initial vertical position of the "levers" as measured by the Scentris; all subsequent deflections are relative to this value. 
In Fig. 6, this experiment is repeated several times; the signals are repeatable and reversible.

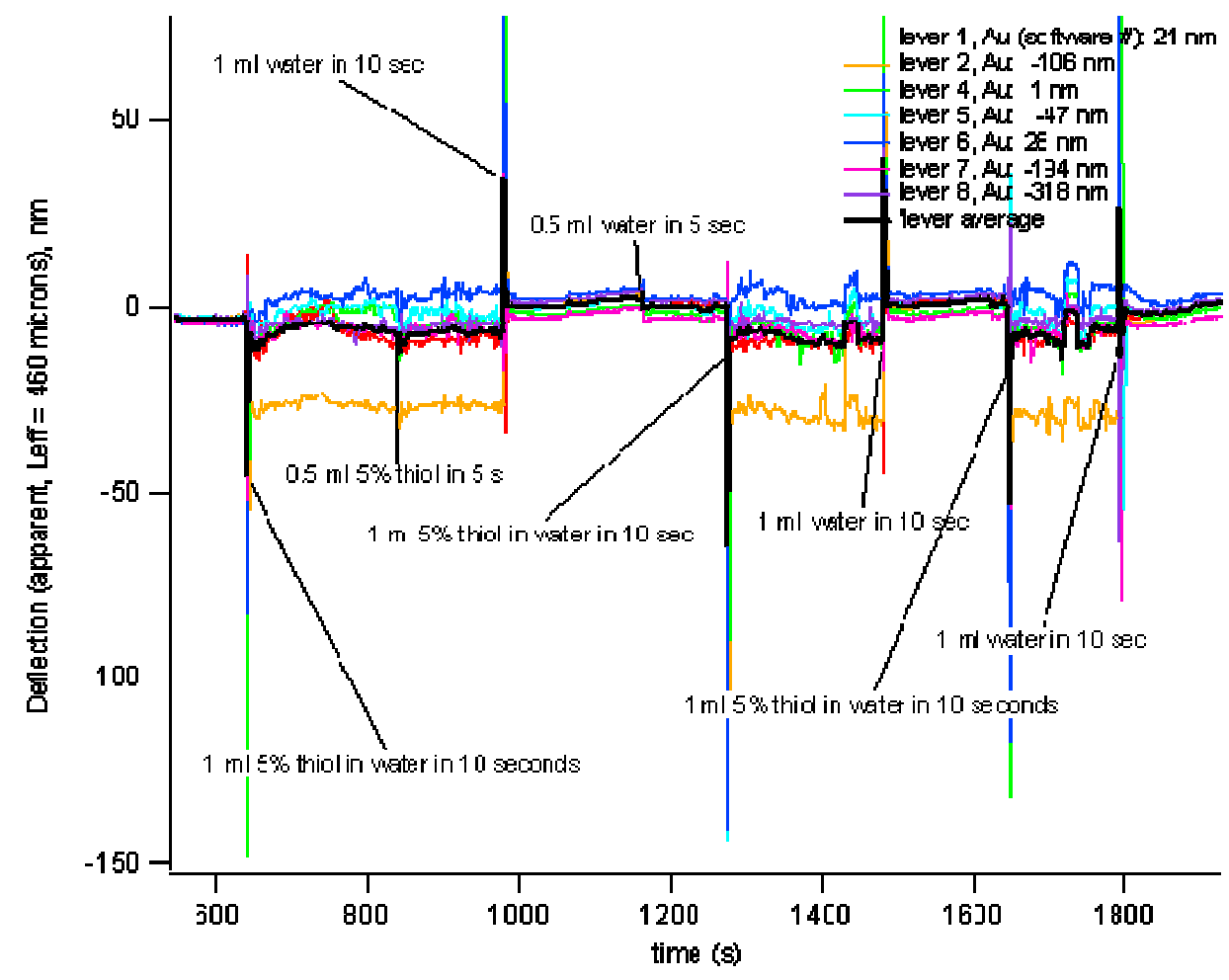

Fig. 6: 5\% mercaptoethanol in water causes apparent deflections in optical measurement system that are repeatable. 
In a second test, pure ethanol (refractive index: 1.36) was flowed into the cell. This caused causes longer-term transients (possibly due to incomplete mixing/exchange in the flow cell) and somewhat larger apparent deflections for some levers, as shown in Fig. 7.

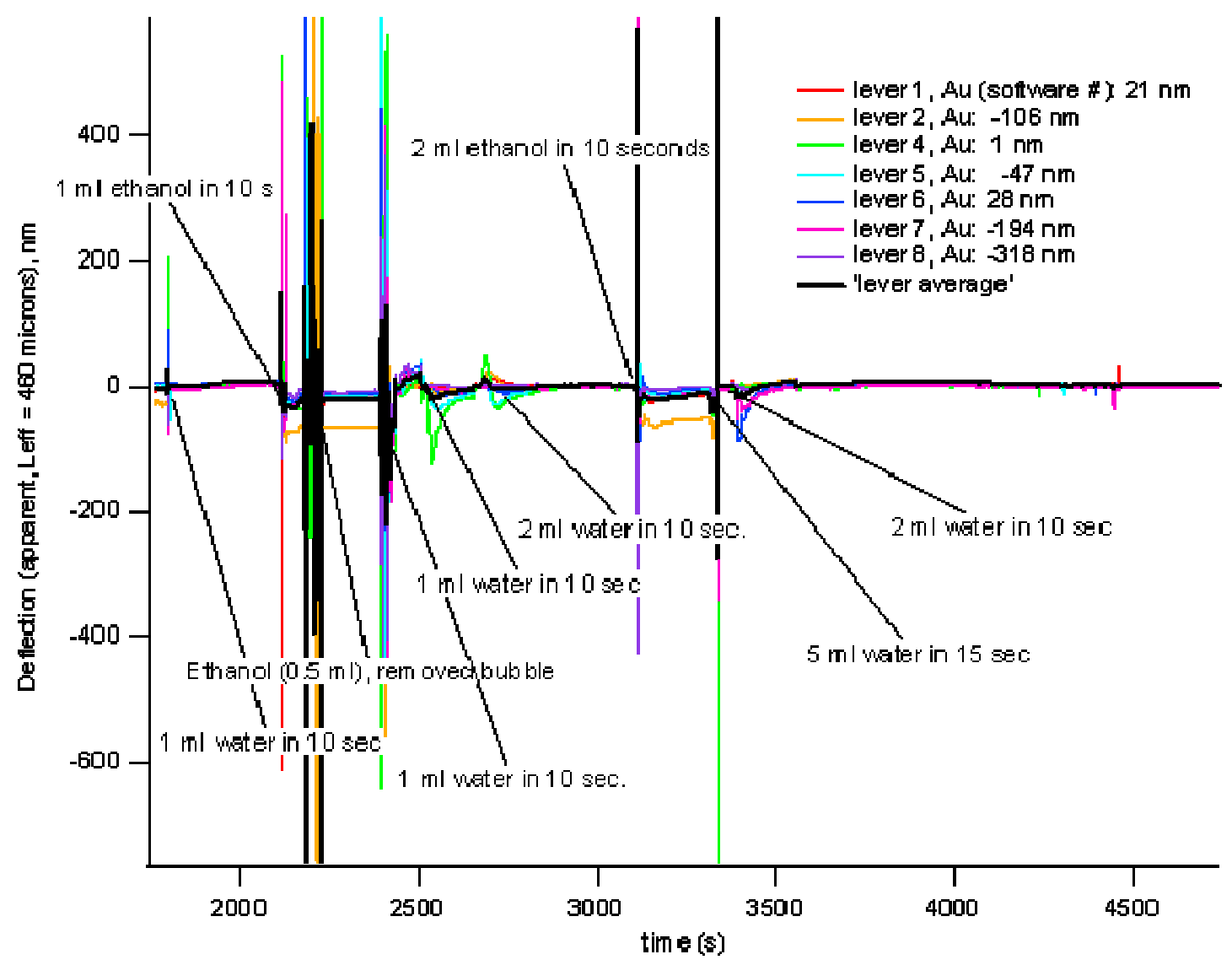

Fig. 7: Longer transients and larger apparent deflections (up to $~ 50 \mathrm{~nm}$, downward) for some levers occur when pure ethanol replaces water in the flow cell. 
Finally, it was observed that the optical deflection system is sensitive to ambient light, even with the opaque hood in place over the test volume. Fig. 8 shows the effects of turning on and off the fluorescent room lights.

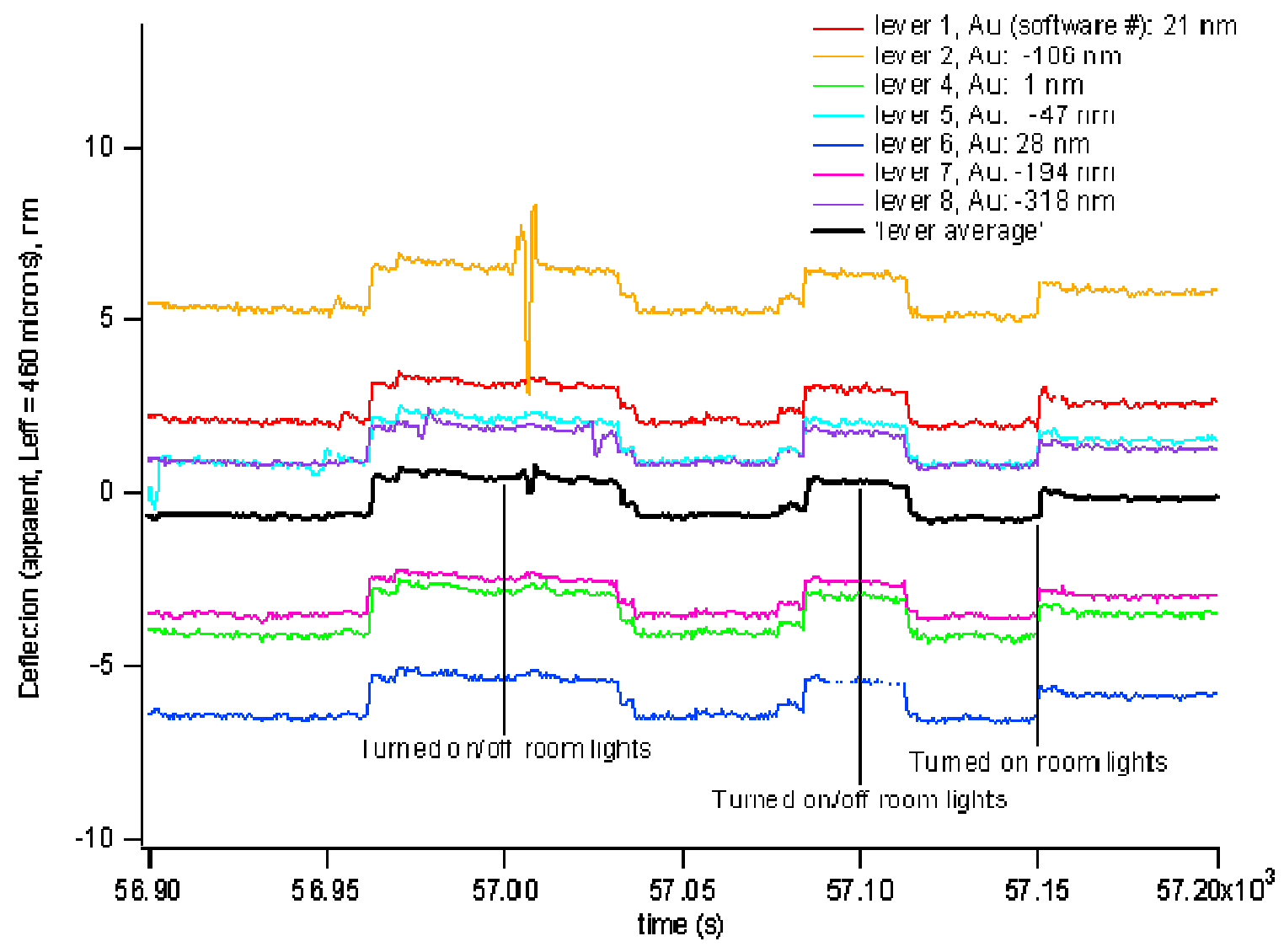

Fig. 8: Small apparent deflections occur when room lights are turned on and off, even with opaque hood in place over test volume. 


\subsection{Experimental description: deflection due to mercaptoethanol}

Experiments were performed with $750 \mu$ m levers, which were coated by evaporation with $1.5 \mathrm{~nm}$ Ti and $20 \mathrm{~nm}$ Au. Levers were cleaned in piranha (3:1 sulfuric acid: hydrogen peroxide) for $\sim 5$ minutes, with gentle agitation, then rinsed in $10 \mathrm{ml}$ water for $\sim 40$ minutes before being loaded directly into the freshly-cleaned flow cell, which was flooded immediately with purified water and sealed.

The effective length of the levers was 520 microns, as shown in Fig. 9. Lever 6 was broken during chip handling.

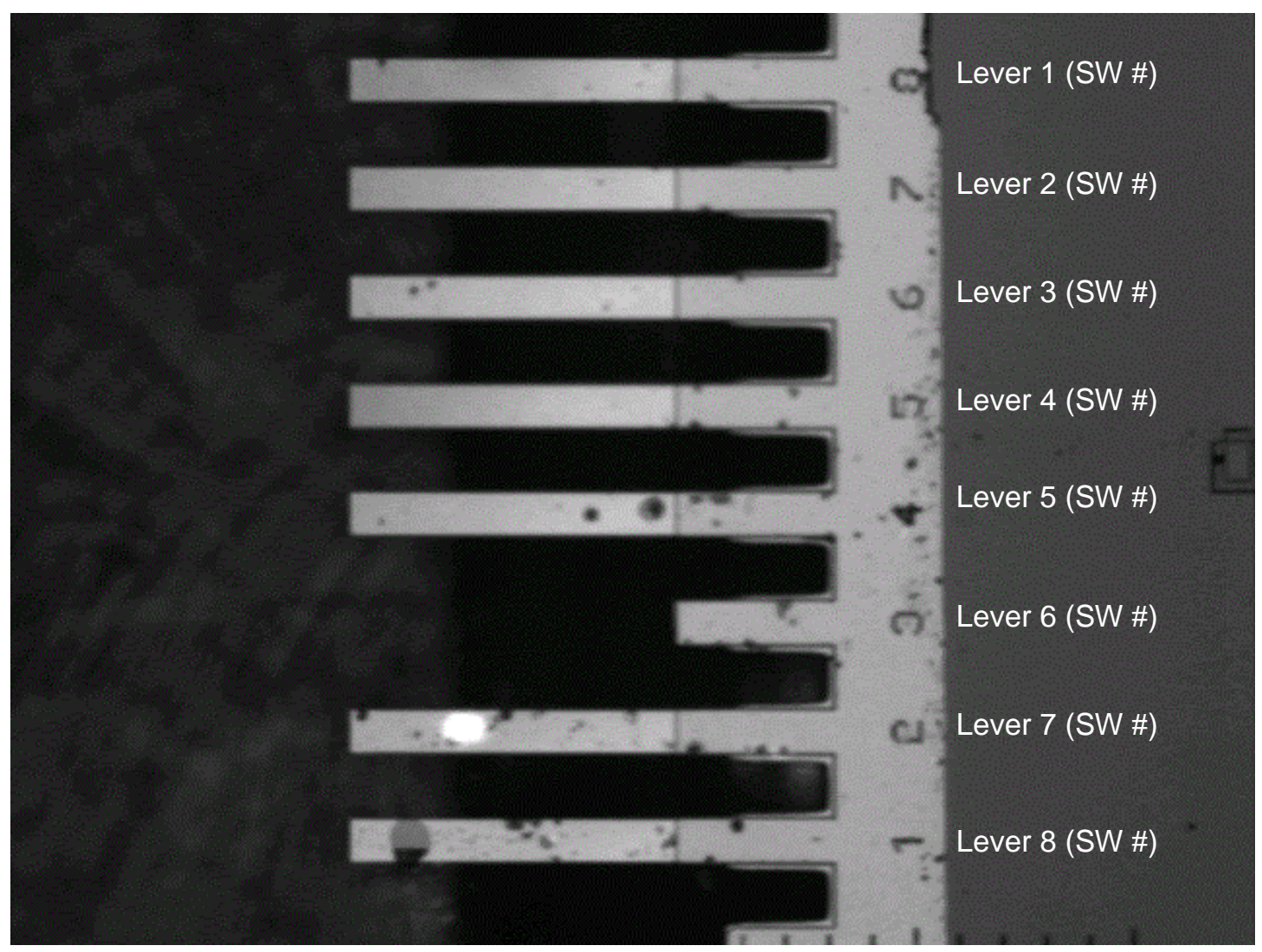

Fig. 9: Scentris chip used for mercaptoethanol desorption experiments.

Constant flow experiments in the 50 microliter cell were performed at 50 microliters/min, alternating purified water and $5 \%$ mercaptoethanol in water. Results of the initial steps in the experiment are shown in Fig. 10. Nanometer values in the legend are the initial vertical position of the levers as measured by the Scentris; all subsequent deflections are relative to this value for each lever. Note that levers with similar initial deflection values tend to drift at similar rates throughout the experiment. The reasons for this behavior are not yet clear; it could be related to the mechanical properties of the levers, or it could be an artifact of the optical detection system. 


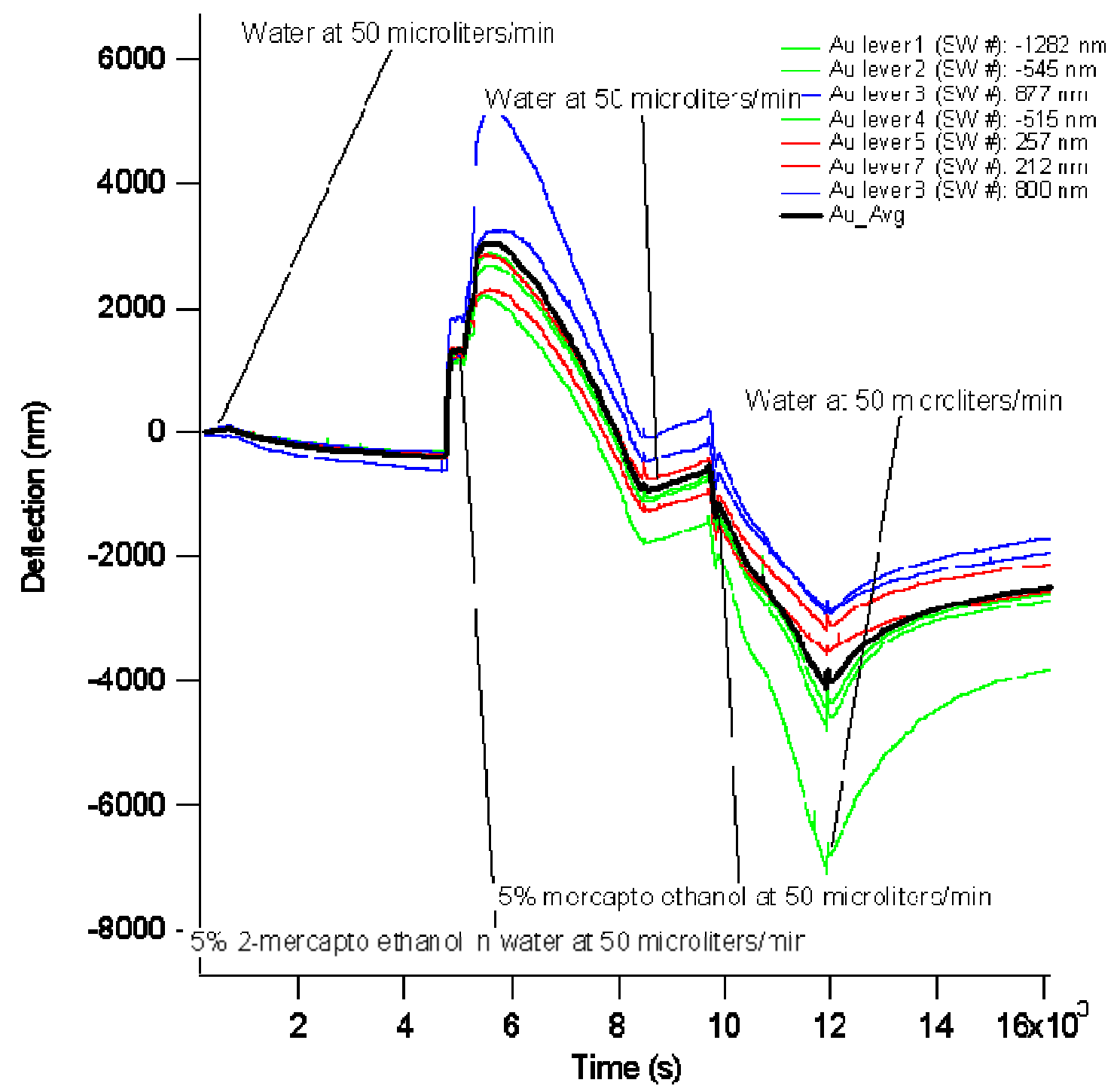

Fig. 10: Initial stages of mercaptoethanol desorption experiment.

An initial positive (downward, away from the Au side) deflection occurs following the first injection of 5\% 2-mercaptoethanol in water. This behavior is consistent with an increase in compressive stress in the cantilevers. This initial positive deflection has been observed repeatedly in previous experiments with mercaptoethanol and Au-coated levers. Subsequent binding of mercaptoethanol to the Au surface induces tensile stresses in the lever, manifested by a negative (upward, toward the Au) deflection of the levers.

When water is flowed through the cell, additional compressive stress is developed in the cantilevers; this is shown in two events at $2200 \mathrm{sec}$ and $12000 \mathrm{sec}$ in Fig. 10. In Fig. 11, the water $/ 5 \%$ mercaptoethanol exchange is repeated twice more, and behavior is consistent: levers bend downward (decrease in compressive stress) when 
mercaptoethanol is introduced, then relax upward (increase in compressive stress) when pure water flows into the cell. Note that the levers never fully relax upward to their initial positions, indicating that significant numbers of mercaptoethanol molecules remain bound to the Au lever surfaces even after rinsing for long periods.

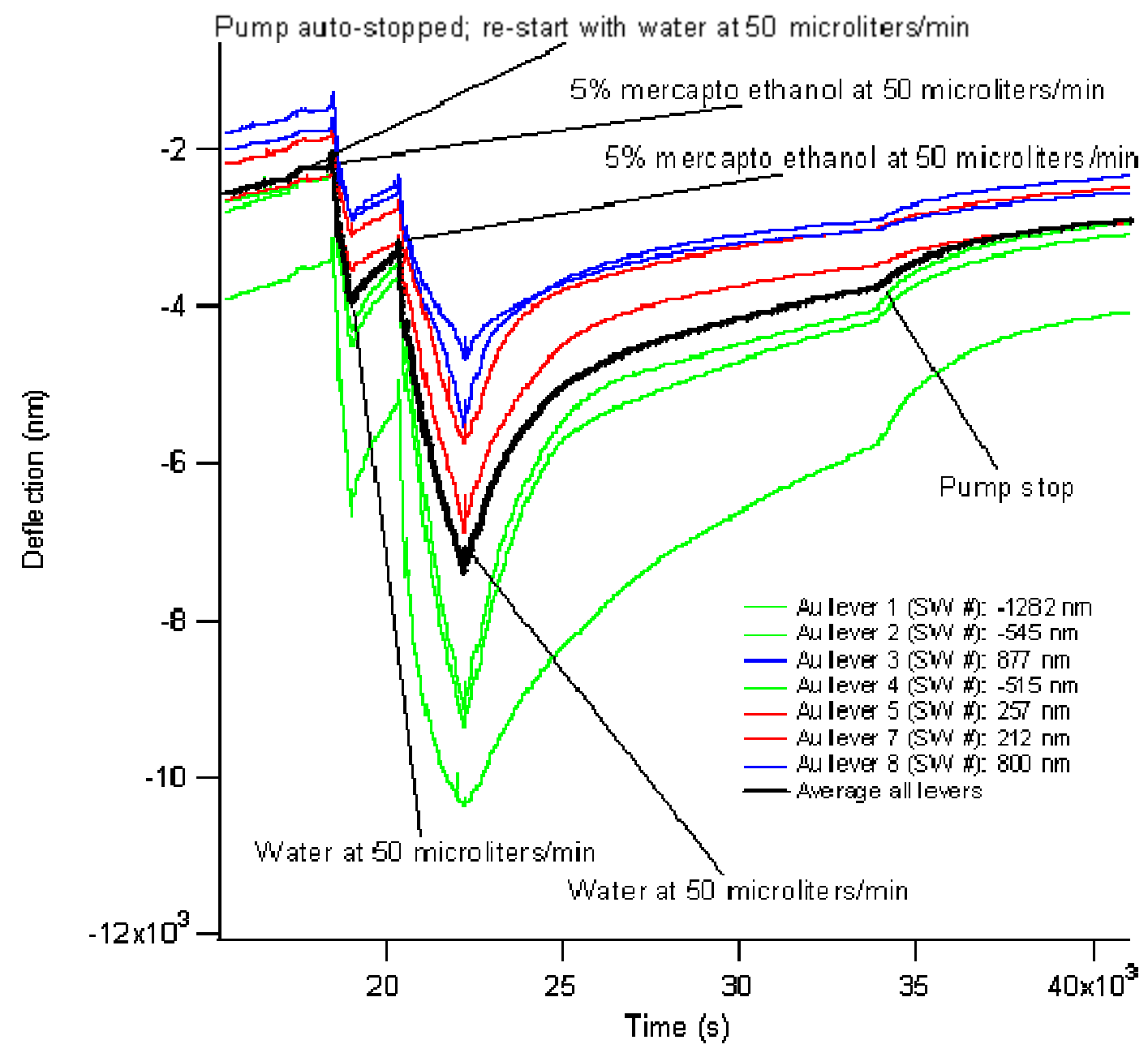

Fig. 11: Final stages of mercaptoethanol desorption experiment. 


\subsection{Experimental description: effects of fluid flow}

750 micron long Au levers (1.5 nm Ti, $20 \mathrm{~nm} \mathrm{Au}$, by evaporation), on the same chip used for the mercaptoethanol experiments previously described, were cleaned in piranha (3:1 sulfuric acid: hydrogen peroxide) for 5 minutes, then rinsed in $10 \mathrm{ml}$ water for 55 minutes. They were loaded directly into freshly-cleaned flow cell, which was flooded with water. The SLD spot positions were set to maintain the levers' effective length near $520 \mu \mathrm{m}$. As can be seen in Fig. 12, some pitting of the Au surface may have occurred since the previous experiment, possibly due to the piranha cleaning process.

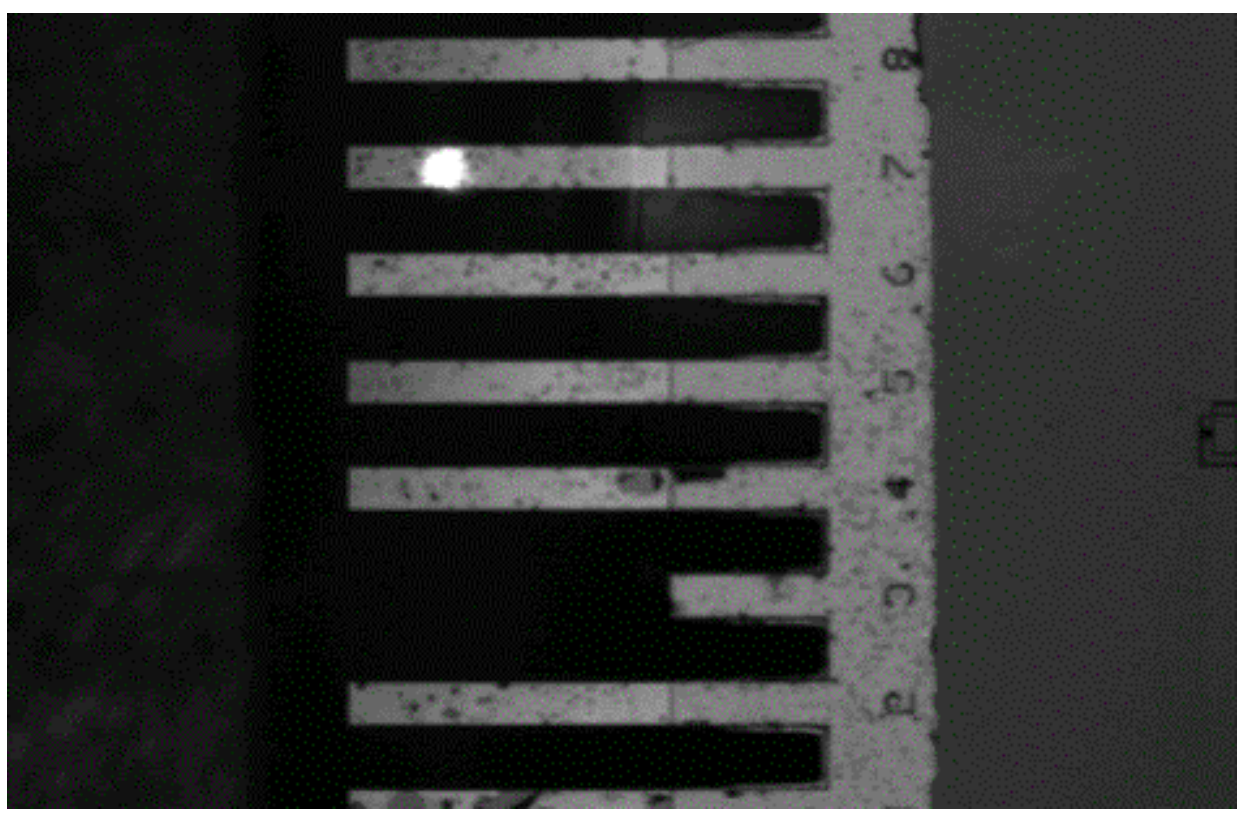

Fig. 12: Levers used for ethanol desorption and flow rate experiments

Constant flow experiments were initially performed in the 50 microliter flow cell. Water and $5 \%$ ethanol in water were exchanged several times. Relative to previouslyobserved deflections induced by $5 \%$ mercaptoethanol in water, the magnitude of these deflections were small, as shown in Fig. 13. The behavior of the levers following each $5 \%$ ethanol injection was consistent and reproducible. These experiments demonstrate that the large deflections observed with the mercaptoethanol in previous experiments were likely caused mainly by molecules adsorbing or desorbing from the surface, as opposed to being caused by changes in water-Au surface interactions mediated by ethanol. 


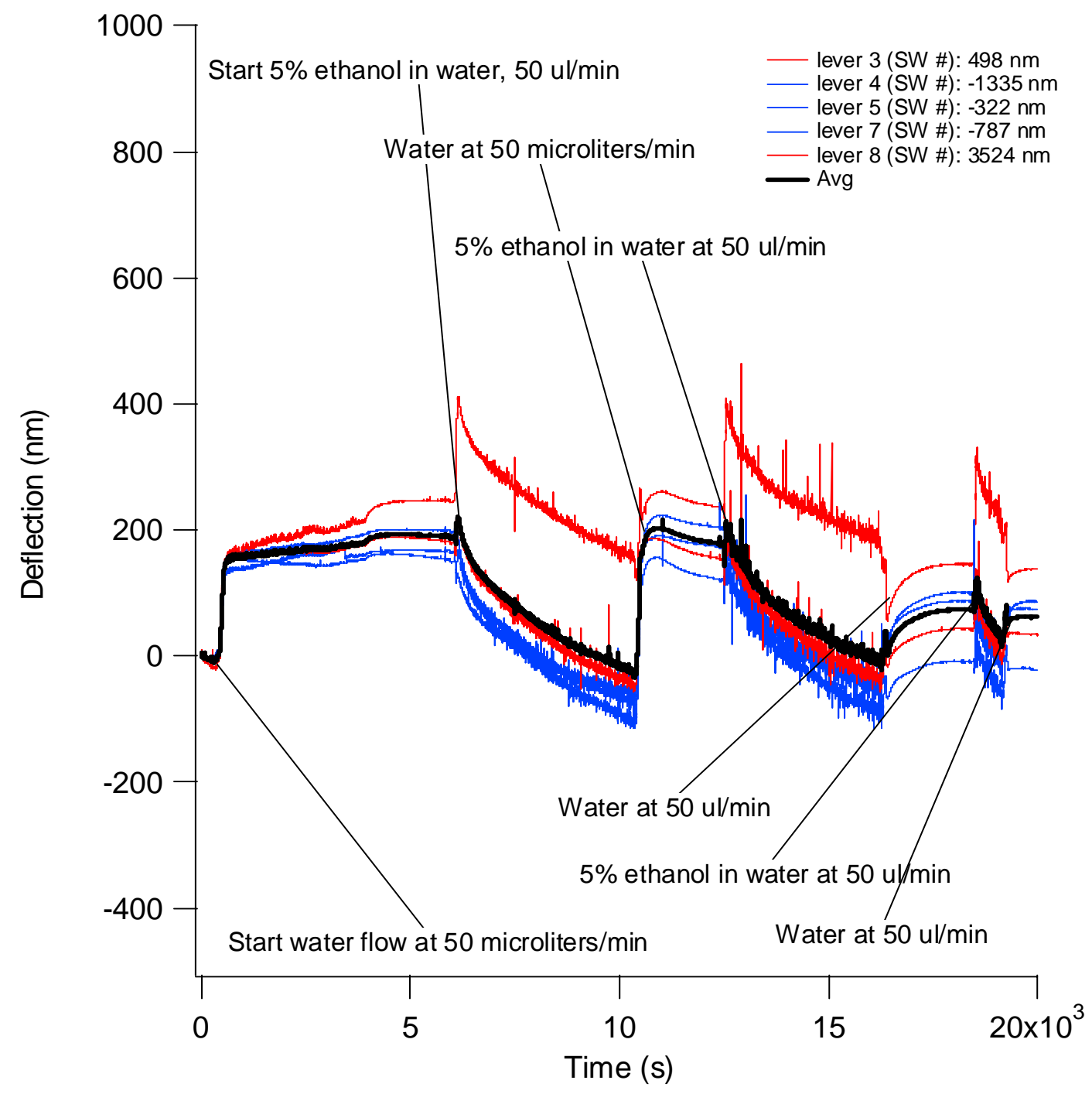

Fig. 13: Constant flow experiments with water and 5\% ethanol in water.

It is interesting to note in Fig. 13 that there is an initial positive deflection of the levers by $175 \mathrm{~nm}$ when flow is initiated at 50 microliters/min. This behavior is important to understand so that flow-induced deflection is not mistaken for deflection induced by chemical changes at the lever surface. Subsequent experiments were therefore performed to learn more about these flow-induced deflections. In Figs. 14 and 15, deflections are shown as the flow rate is slowed from 50 to 2 microliters/min. 


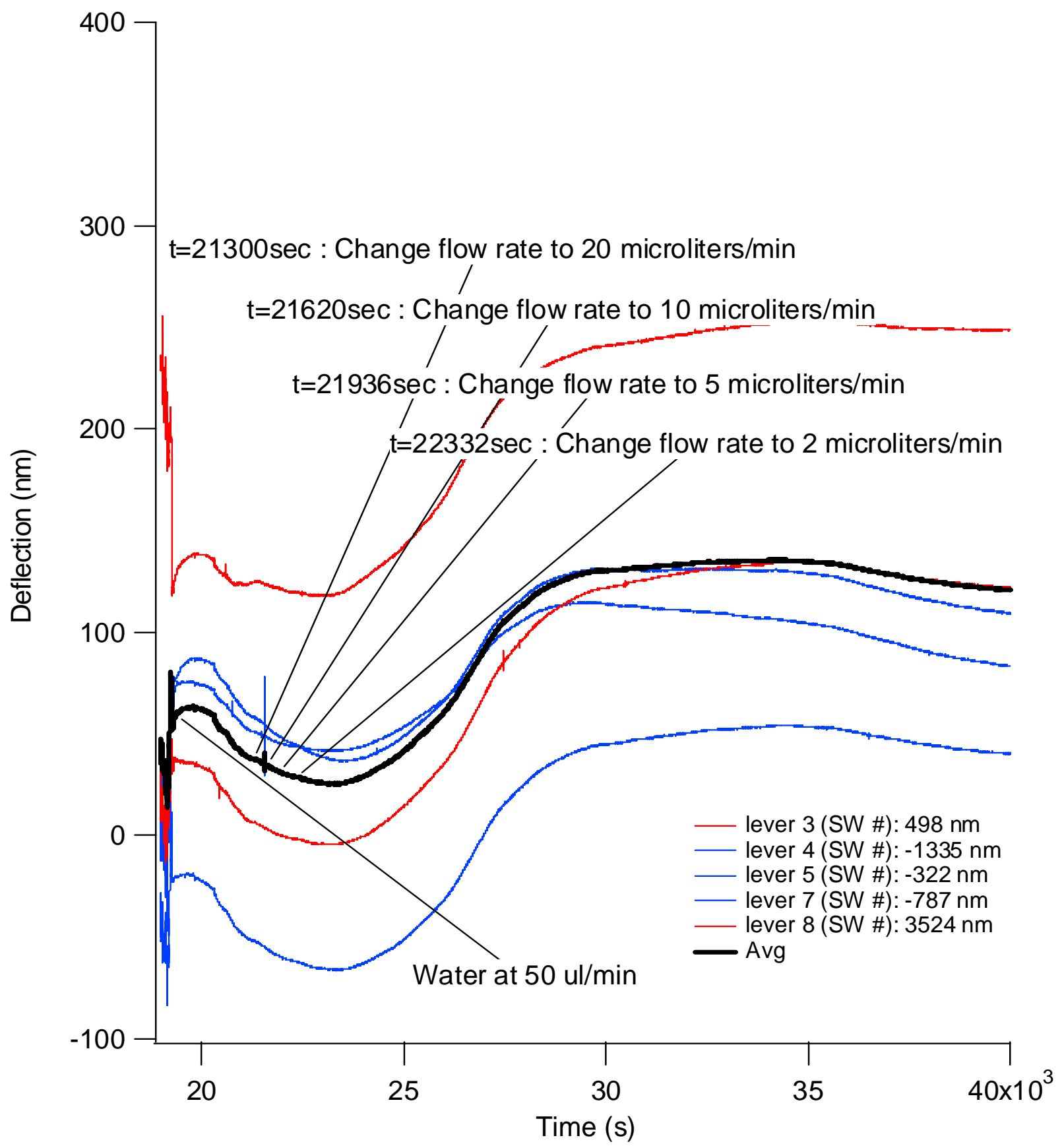

Fig. 14: Effect of reducing water flow rate from 50 to 2 microliters/min.

The levers drift downward, then back up after the flow rate is stabilized at 2 microliters/min for an extended period. Fig. 15 shows an expanded view of the average lever deflection as the flow rate is changed from 20 to 2 microliters/min. 


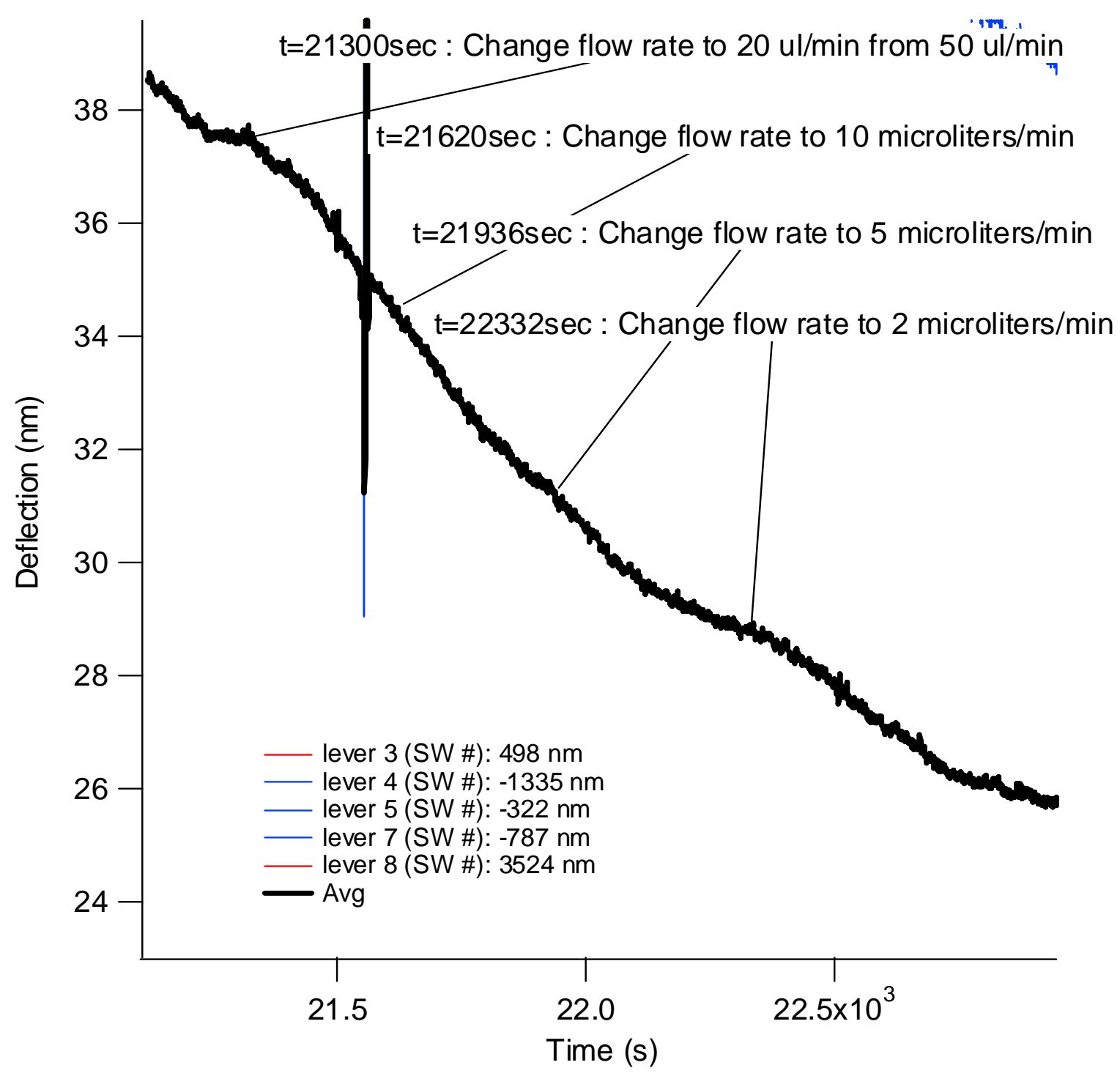

Fig. 15: Average lever deflection as water flow rate is decreased to 2 microliters/min from 20 microliters/min.

In Fig. 16, some deviations occur in the downward drift trend as the flow is reduced, but these are relatively small. Fig. 16 shows the effect of increasing flow rate from 2 to 200 microliters/min, then back to 2 microliters/min. It is difficult to generalize about the direction of deflection induced by increased or decreased flow. Figs 14 and 15 show downward deflection as flow rate is decreased, while Fig 16 shows downward deflection as flow rate is increased. In general, however, larger flow rates do seem to cause larger deflections, and when the flow rate is stabilized for extended periods at low values ( $<5$ microliters/min), the levers tend to drift upward for this experimental set. 


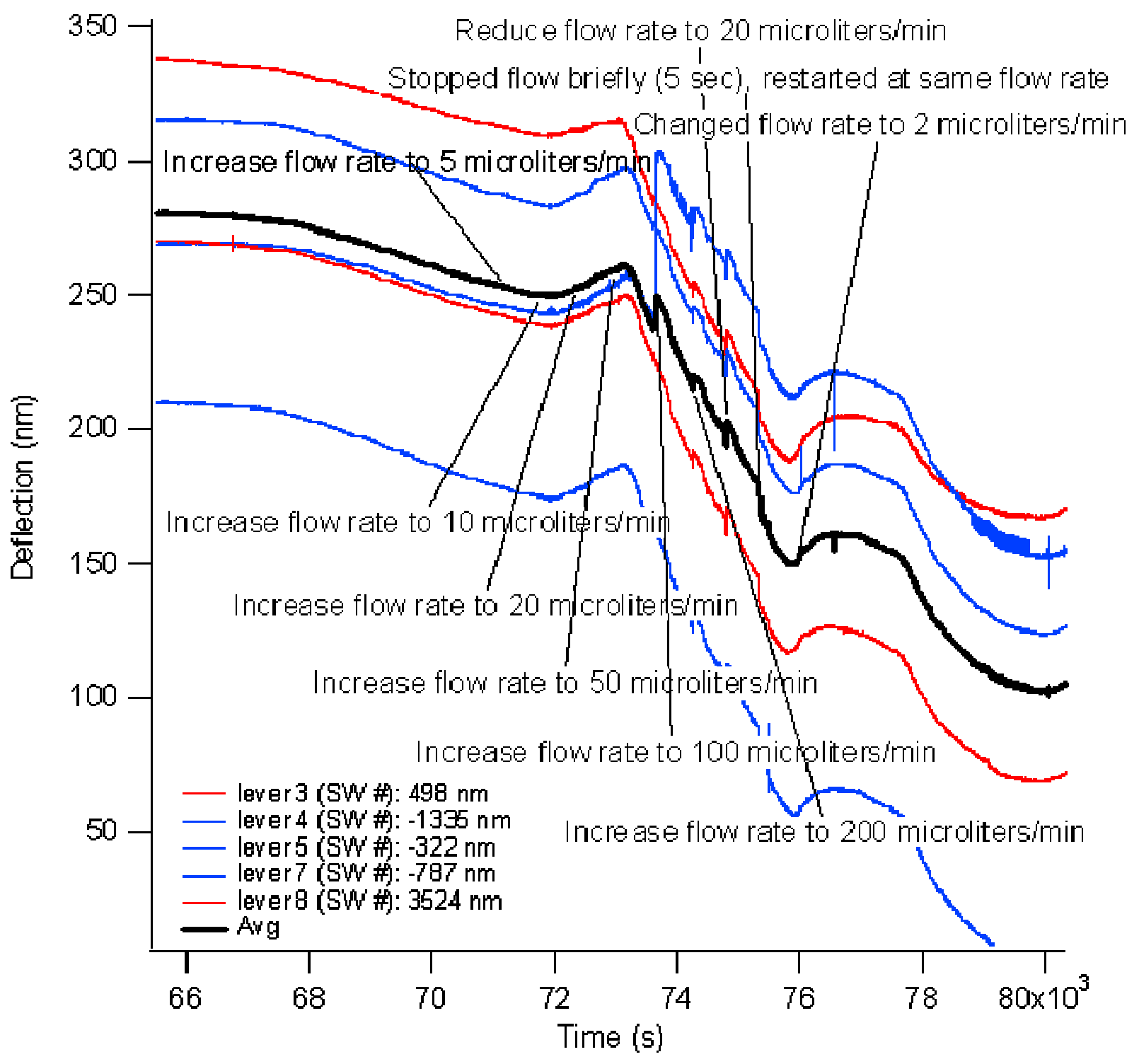

Fig. 16: Lever deflections as water flow rates are changed.

Fast injection conditions are of particular interest for detection experiments. Ideally, chemicals can be introduced rapidly into the fluid cell so that the resulting behavior of the levers can be characterized starting at a single time (as opposed to the case when chemicals are slowly flowed into the cell over minutes or hours). Also, since high flow rates themselves can cause lever deflections, the ideal chemical detection experiment would occur under low- or no-flow conditions. Data in Fig. 17 show the results of changing flow rates briefly from very low levels ( 2 microliters/min), briefly to high levels for injection (100 or 200 microliters/min), then back to low levels for detection. 


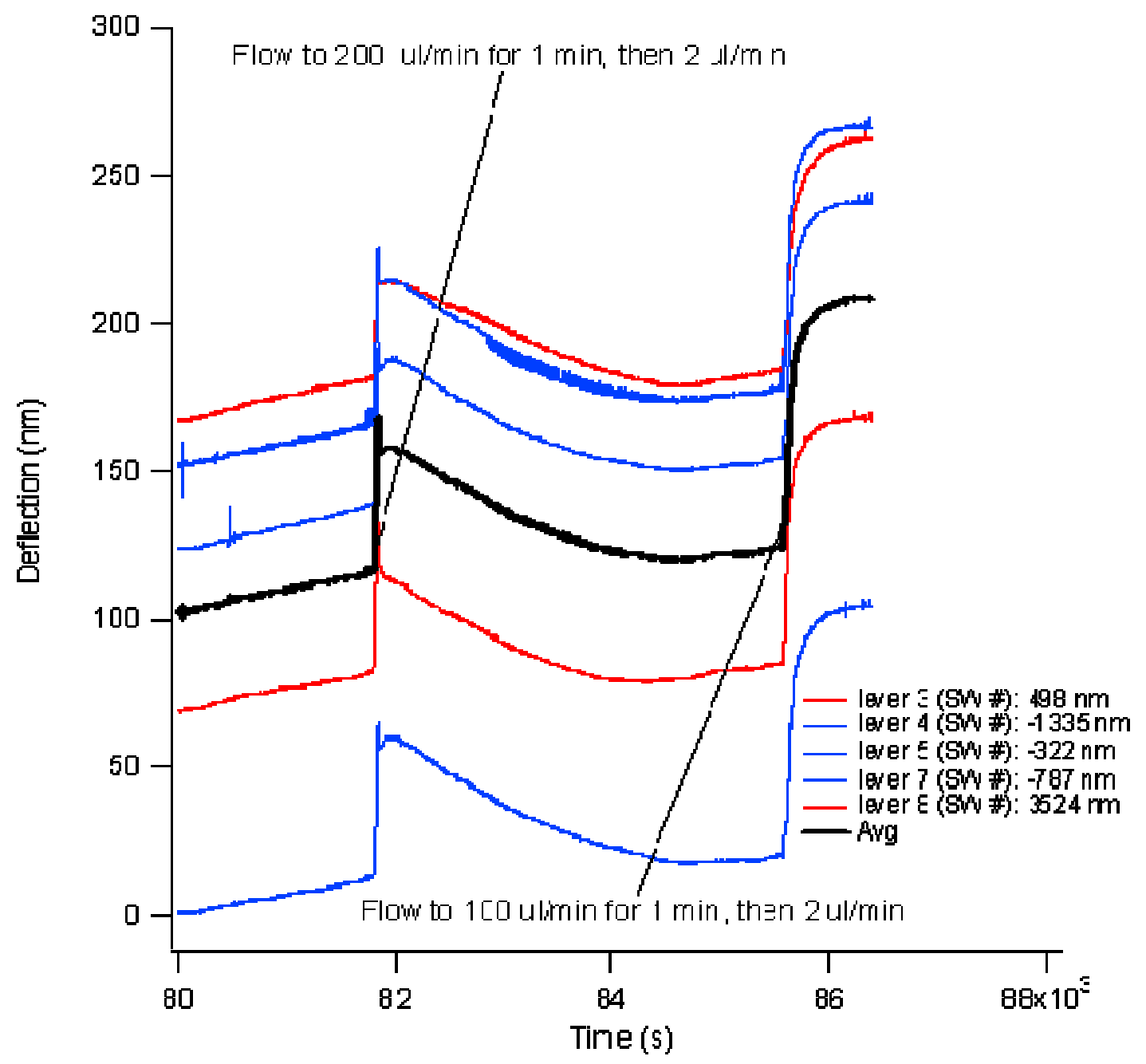

Fig. 17: Effect of increasing water flow rates briefly for simulated injection of a chemical of interest into the flow cell; the initial flow rate is 2 microliters/min.

As can be seen in Fig. 17, increasing flow rates to 100 or 200 microliters/min from 2 microliters/min causes a large, immediate upward deflection. More problematic for detection experiments, is that the fast changes can cause extended relaxation periods (1000's of seconds) during which the levers drift significantly. 


\subsection{Experimental description: temperature and chemical effects}

To calibrate the deflection of Scentris cantilevers, it is helpful to use a temperature stimulus which can be measured independently. As will be described in Section 4, the spring constant of the cantilever can be calculated based upon the known dimensions of the cantilever and the observed deflection response of the cantilever to an independently measured temperature increase. As can be seen in Fig. 18, critical dimensions of the cantilever can be determined by scanning electron microscopy (SEM).

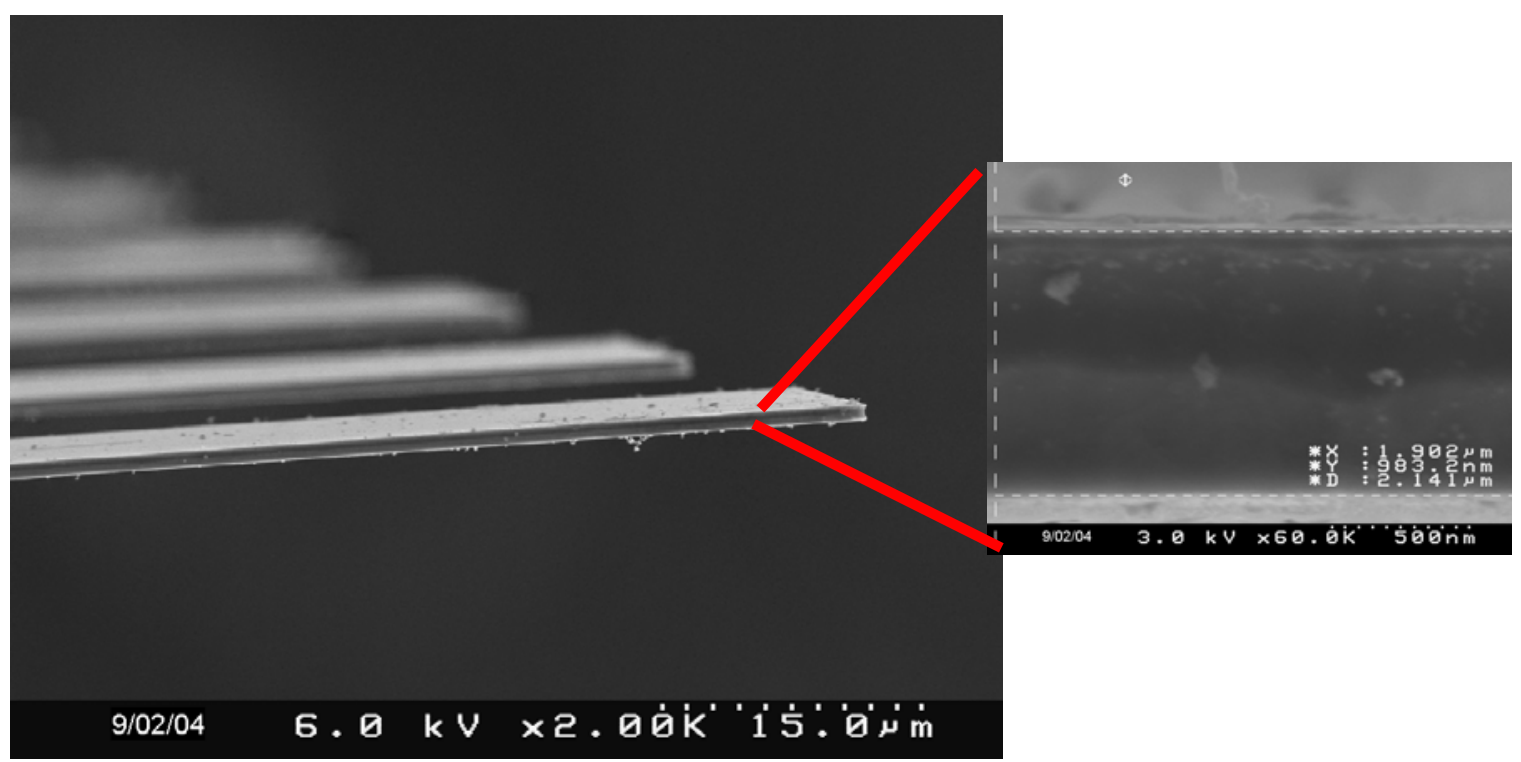

Fig. 18: SEM image of cantilevers showing sensitive measurement of the cantilever thickness.

Here, we describe a calibration experiment in which 500-micron long, Au-coated levers were exposed to a temperature pulse, then a chemical stimulus in the form of a $1 \%$ (by volume) concentration of mercaptoethanol in pure water, and finally a second temperature pulse.

Levers were coated with $20 \mathrm{~nm}$ of evaporated $\mathrm{Au}$, and cleaned as described previously with piranha immediately before being loaded into the water-filled 50 microliter flow cell. Except for brief pauses to exchange syringes in the syringe pump, the entire experiment was performed at a constant flow rate of 20 microliter/min. The results of the experiment are shown in Fig. 19. 


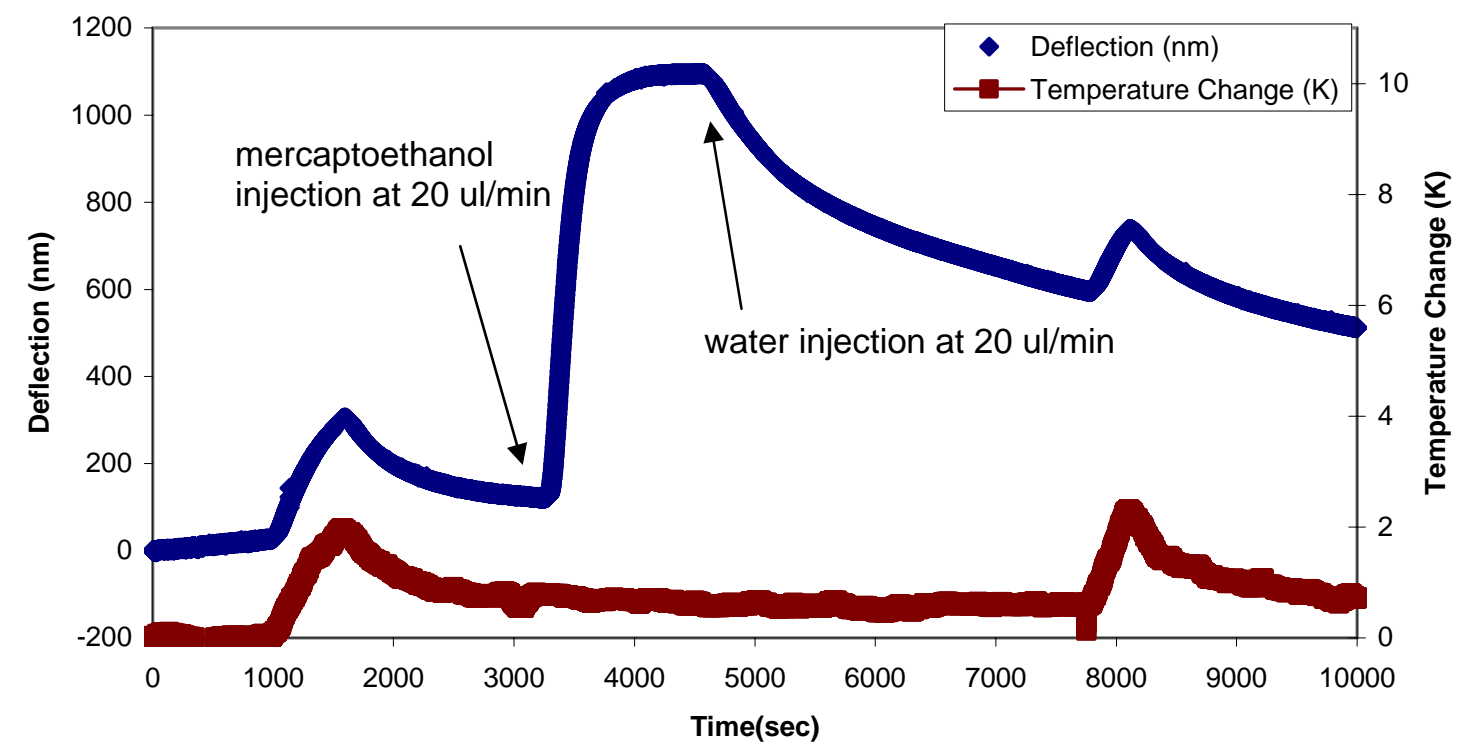

Fig. 19: Deflection of cantilever due to temperature and chemical stimuli.

The results of this experiment will be discussed in detail in Sections 3-5, where the deflections are modeled and quantitatively analyzed to extract chemical parameters, such as the absorption constant, that are necessary for detection applications.

In order to ensure that experimental results are not being affected by uncontrolled variables, and that modeling assumptions are realistic, a large data set was obtained with various concentrations, target molecules and solvents. The experimental parameters of these experiments are summarized in Table 1 , and representative results of the experimental data set are shown in Figs. 20 and 21.

Table 1: Number of experiments for several experimental conditions:

\begin{tabular}{|l|l|l|l|l|}
\hline Solvent: & & Water & Ethanol & Ethanol \\
\hline Solute: & & Mercaptoethanol & Mercaptoethanol & Hexadecane \\
\hline Concentration (M): & $1 . \mathrm{E}-04$ & 0 & 0 & 2 \\
\hline & $1 . \mathrm{E}-03$ & 0 & 6 & 3 \\
\hline & $1 . \mathrm{E}-02$ & 1 & 1 & 0 \\
\hline & $1 . \mathrm{E}-01$ & 0 & 3 & 0 \\
\hline & $1 . \mathrm{E}+00$ & 3 & 2 & 0 \\
\hline
\end{tabular}




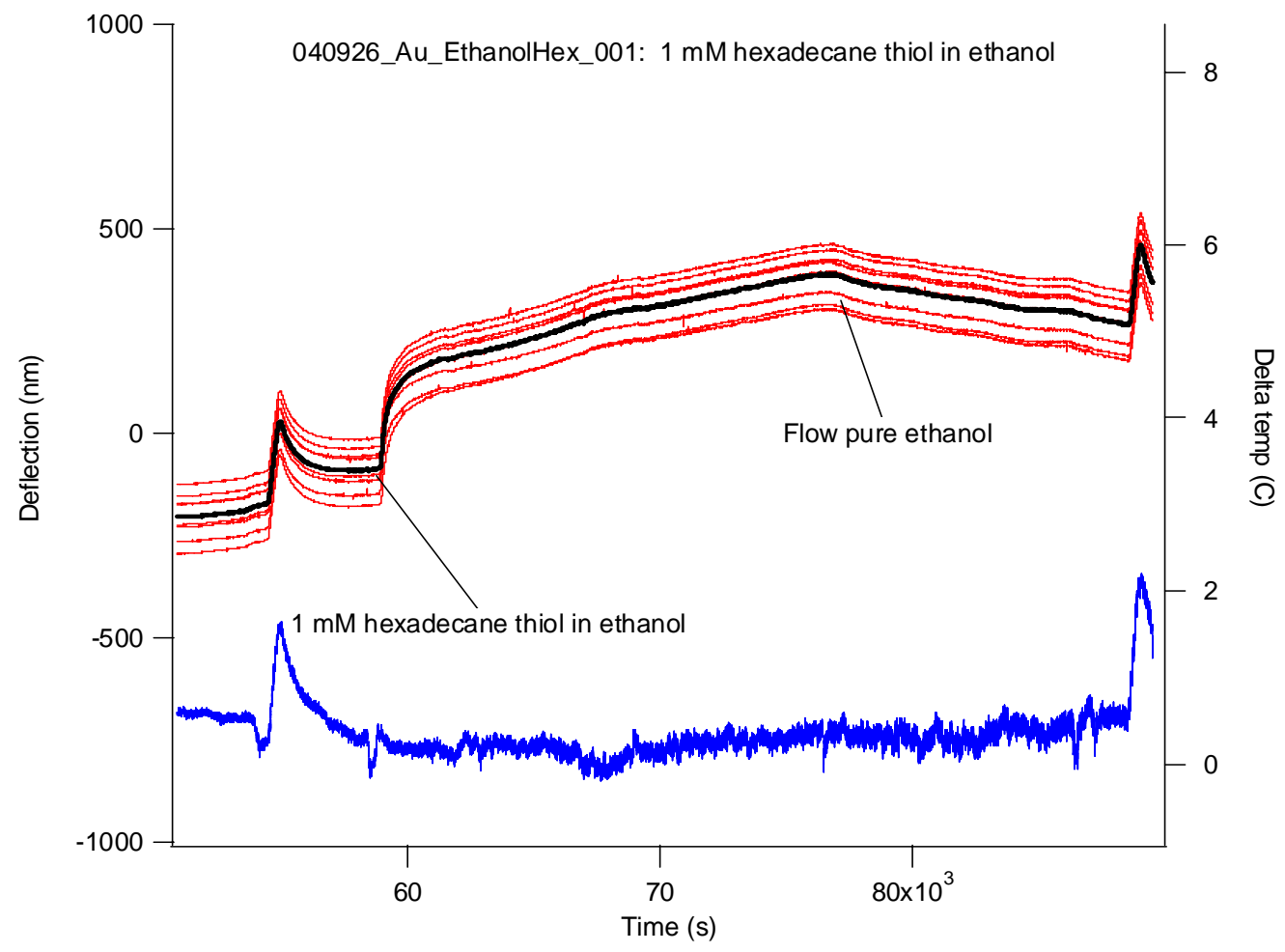

Fig. 20: Experimental results for $1 \mathrm{mM}$ hexadecane in ethanol.

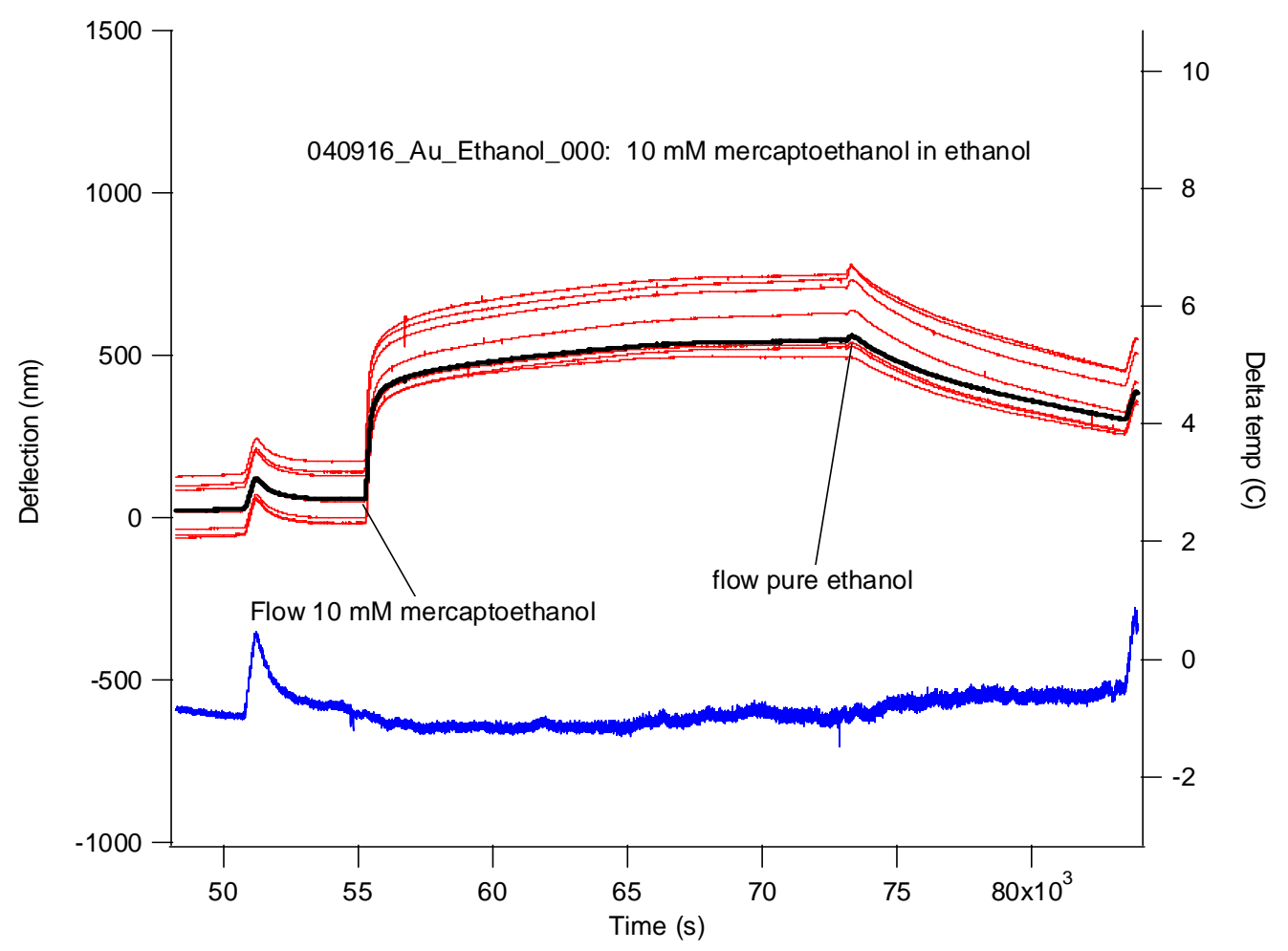

Fig. 21: Experimental results for $10 \mathrm{mM}$ mercaptoethanol in ethanol. 


\section{Physical chemistry}

In this section, we describe how we predict changes in surface stress as a function of surface loading. The overall analysis in the modeling begins with Stoney's equation (Eq. 4), which states that the deflection of the cantilever is directly proportional to the difference in surface stress on the cantilever surface. The physical system is shown below in Fig. 22.

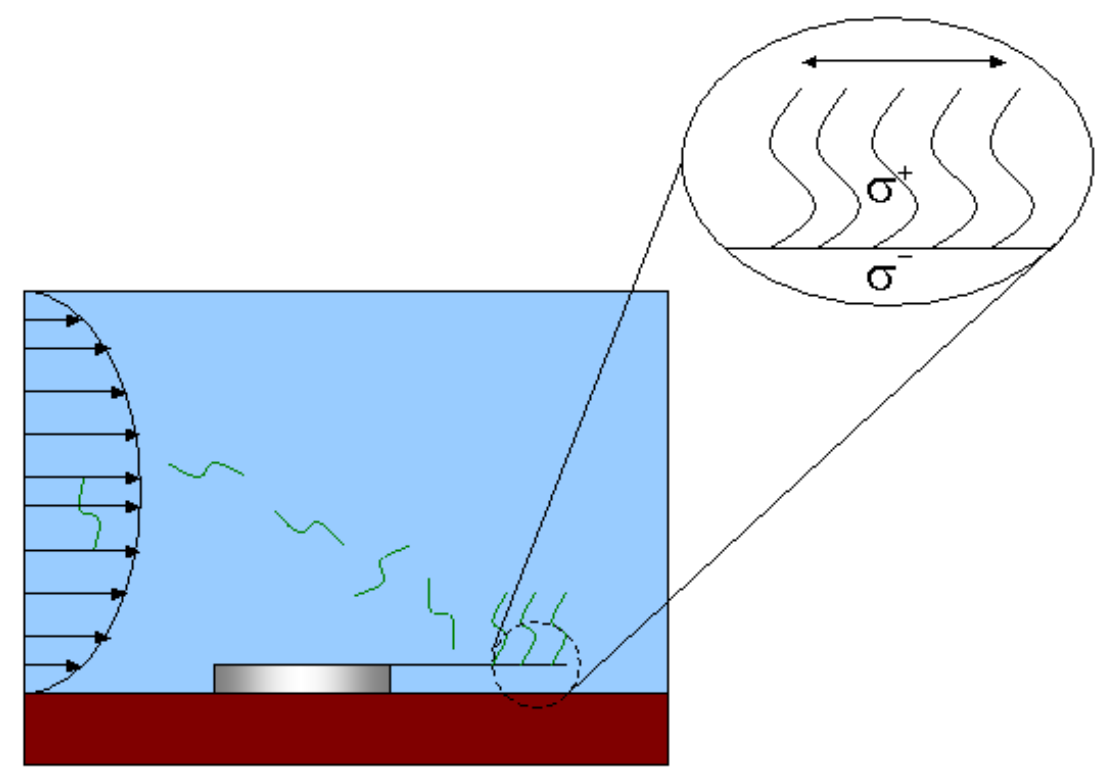

Fig. 22: Flow over a cantilever with polymer, green species, depositing on the surface of a cantilever. The deposited polymer self-assembles and the assembled species induces a lateral stress that causes a stress imbalance between the top and bottom surfaces, i.e., $\sigma^{+}$ and $\sigma^{-}$.

This stress differential constitutes the signal. We follow the approach as described by Lavrik et al. [3] to relate the surface stress difference to the surface coverage and the free energy of adsorption (Eq. 2). What remains is to develop physical models for $\Gamma(\mathrm{t})$ and $\Delta \mathrm{G}$.

To estimate the rate of change of surface concentration, $\Gamma(\mathrm{t})$, we employ a modified form of Eq. 3 by re-defining a rate of desorption, $k_{d}^{\prime}$, where $k_{d}^{\prime}=k_{d} \Gamma_{\max }$. With this new definition, Eq. 3 becomes: 
Eq. 5

$$
\frac{d(\Theta)}{d t}=k_{a} c(t)(1-\Theta)-k_{d}^{\prime} \Theta
$$

All terms are as described above in Eq. 3 with the exception of $k_{d}^{\prime}$. Note that Langmuir kinetic equations have been presented in various forms in the open literature. The above form, Eq. 5, was chosen to enable a one to one comparison between rate constants predicted by the MBP presented here and those reported in the literature [4,5,6]. Eq. 3 above, however, is based on the rigorous analysis presented in Appendix A.

In solving the Langmuir kinetics, we hold the concentration as a constant in Eq. 3 and then build in the dynamic nature of the signal through the solution to a decoupled differential equation for the dynamics of the bulk phase signal concentration (see Eq. 7). This is justified since the time scales between surface adsorption/desorption and bulksignal concentration are sufficiently disparate.

Once the input signal, $c_{0}$, is turned off we again fit the desorption process with an $\mathrm{n}^{\text {th }}$ order Langmuir desorption mode:

Eq. 6

$$
\frac{d(\Theta)}{d t}=-k_{d}(\Theta)^{n}
$$

The fit to the actual desorption data revealed a third-order dependence on the dimensionless surface concentration, or $n=3$. Desorption processes are typically modeled with a first or second order model to extract $k_{d}$; however, in our case, the best fit was with a third order model; consequently, the third order dependence on dimensionless surface concentration was not expected. This fact that the best fit for the desorption behavior was with $n>1$ suggests that there may be a distribution of activation energies associated with the desorption process (see Appendix A).

To account for the dynamics of the system, more specifically the transient nature of the system owing to fluid flow rates, we developed a dynamic estimate of the bulk species concentration based on a modified stirred tank reactor system, see Fig. 23 below. This mathematical description of the flow cell is a useful approximation for observing time-dependent fluidic behavior, but experimental evidence suggests that at the low flow rates applied in the $50 \mathrm{ul}$ flow cell experiments described here (50 microliters/min and smaller), flow is in fact relatively laminar. Levers on the outside of the array, for example, were consistently observed to react to chemical signals a few seconds before the inner levers. If the system acted as a true stirred tank, all levers would react to chemical stimuli simultaneously. 


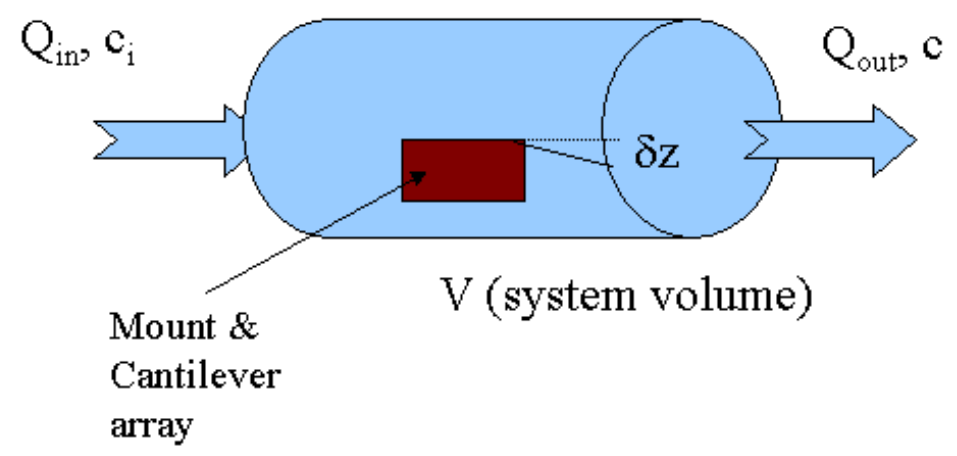

Fig. 23: Model flow cell for cantilever array. $Q_{\text {in }}$ and $Q_{\text {out }}$ are the volumetric flow rates in and out of the cantilever array chamber. $c_{i}$ and $c$ are the input concentration and the concentration within the volume for perfect mixing.

The resulting equation we used to predict the bulk, target species concentration was the following form:

Eq. 7

$$
c(t)=c_{0}\left\{\left(1-e^{-\left(t-t_{O N}\right) / \tau}\right) H\left(t-t_{O N}\right)-\left(1-e^{-\left(t-t_{O N}\right) / \tau}\right) H\left(t-t_{\text {OFF }}\right)\right\}
$$

Here $c(t)$ is the time dependent concentration of our target species, $c_{i}$, is the input signal or concentration, ton is the time at which the signal is turned on and tofF is the time when the signal is turned off and $\tau, \tau=\frac{V}{Q}$, is the residence time of the fluid in the cantilever array volume, $V . \mathrm{H}(\mathrm{t})$ here is the Heaviside step function; i.e. the function that is zero when the argument is negative and one when it is positive. For the given system and using half of the chamber volume, $\tau \approx 75 s$, and the resulting response or concentration in the chamber, for the gross approximation of perfect mixing, is shown below in Fig. 24. 


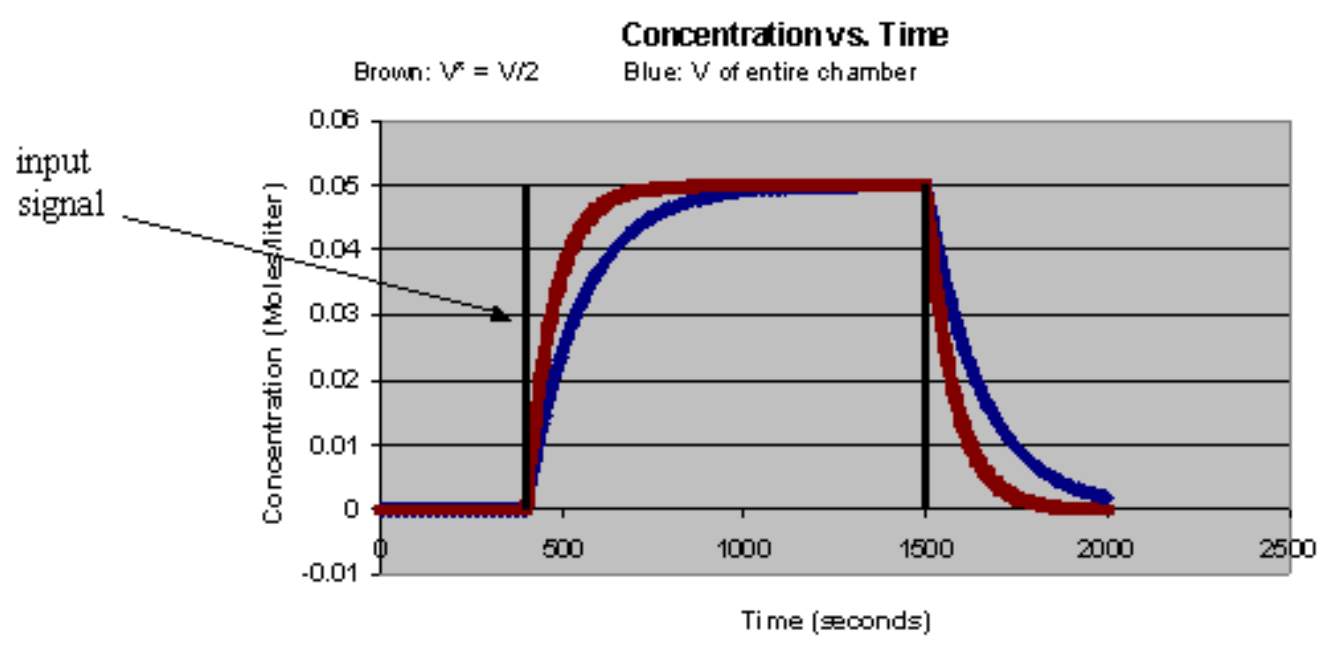

Fig. 24: Bulk concentration in cantilever array flow chamber. Given that the cantilever array is at the center of the chamber, predictions were made for the entire chamber volume, blue curve, and for half of the chamber volume, brown curve. The broad black line represents the time on and the time off of the signal.

All of these features were built into the model based signal processor (Section 5), and the signal based processor/estimator was used to fit these coupled equations predict the optimal values for $k_{a}, k_{d}$ and $\Gamma_{\max }$. With these parameters specified, we calculate the free energy for adsorption, $\Delta G=-R T \ln \left(\frac{k_{a}}{k_{d}}\right)[4,6]$. For any target species, we can then fit to get the appropriate adsorption parameters. Furthermore, we also built in the cantilever response to thermal effects, or coefficient of thermal expansion effects, as will be described in Section 4.

\section{Mechanical modeling}

In this section we describe the modeling of micromachined cantilevers using finite element methods and closed form analytical expressions. The results are applied to calibrate the cantilevers based on their response to known temperature stimuli.

\subsection{Finite element model of a complete array}

A finite element model of an eight-cantilever array was created to conduct simulations. The wafer, cantilevers, and anchors are represented by eight-node solid elements. The gold coating on the cantilever surfaces are represented by four-node membrane (shell with one integration point) elements. The boundary conditions are fixed (no displacement, no rotation) on the bottom surface of the chip. This will account for compliance at the root of the cantilevers. 
Using this model, various loading conditions can be applied, e.g., fluid interaction, heat transfer, applied surface traction. Dynamic behavior can then be simulated using the 3D nonlinear, implicit finite element code, NIKE3D. The model is sufficiently generalized to account for variations in geometry, material properties, and initial stress.
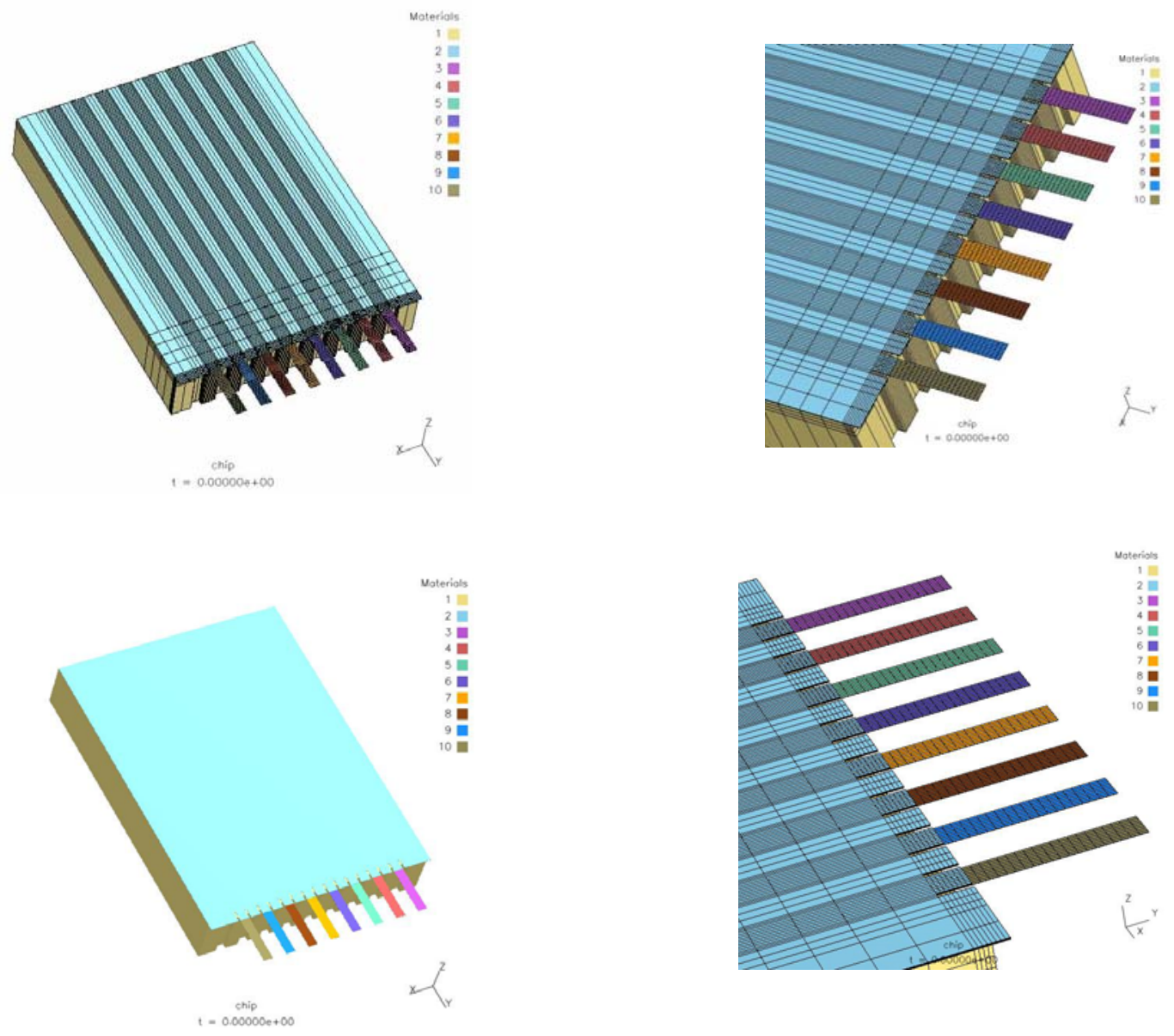

Fig. 25: Finite element mesh of an IBM Zurich Research A1: Water, cantilevers, and anchor.

\subsection{Finite element model of a single cantilever}

A finite element model was created to focus on the behavior of a single cantilever. As in the full array model, the cantilever, anchor, and a portion of the wafer are represented by eight-node solid elements. The gold coating on the cantilever is represented by four-node plate elements. In this case, only membrane behavior is captured by using one integration point with the shell elements.

Two mechanisms which cause cantilever deflection were studied with the model. The first is temperature-dependent thermal expansion. The cantilever deflects in the same fashion as bi-metallic beams. As the temperature changes, the silicon and gold expand at 
different rates (as described by the thermal expansion coefficient). This causes a stress in the beam resulting in a deflected equilibrium shape.

The second mechanism is a chemically induced stress which also causes the cantilever to deflect to maintain equilibrium. Chemical adsorption/desorption in the gold layer causes a change in the free energy of the gold layer. This results in an applied traction to the top surface of the silicon cantilever. To maintain static equilibrium the cantilever must change its shape. Due to the beam-like geometry of the cantilever, the deflection is predominately a displacement in the direction normal to the cantilever's surface (z direction in Fig. 26).

This effect cannot be captured directly in the current version of NIKE3D, However, it can be included by an equivalent thermal stress in fictitious shell elements. Those shell elements are given properties so that they do not contribute to the mass and stiffness of the cantilever and that plane stress arises from contributions from thermal expansion but not bending, i.e., the thermal expansion coefficient, $\alpha$, is very large relative to the Young's modulus. From the plane stress equation,

Eq. 8

$$
\sigma_{x x}=\frac{E}{1-v^{2}}\left(\varepsilon_{x x}+v \varepsilon_{y y}\right)-\frac{E}{1+v} \alpha \Delta T
$$

if $\alpha$ is very large, then $\sigma_{x x}$ is dominated by the second term on the right hand side. In these equations, E and $v$ are the Young's modulus and Poisson's ratio for Si, respectively. $\Delta T$ is the change in temperature. $\varepsilon_{x x}$ and $\varepsilon_{y y}$ are the in-plane strains.

Thermo-elastic material properties (Material Type 4 in NIKE3D) are taken to be for silicon: $E=150 \mathrm{e} 9 \mathrm{~kg} / \mathrm{m}^{3}, \mathrm{v}=0.2$, and $\alpha=4.2 \mathrm{e}-6 / \mathrm{K}$ and for gold: $E=80 \mathrm{e} 9 \mathrm{~kg} / \mathrm{m}^{3}, \mathrm{v}$ $=0.42$, and $\alpha=14.2 \mathrm{e}-6 / \mathrm{K}$.

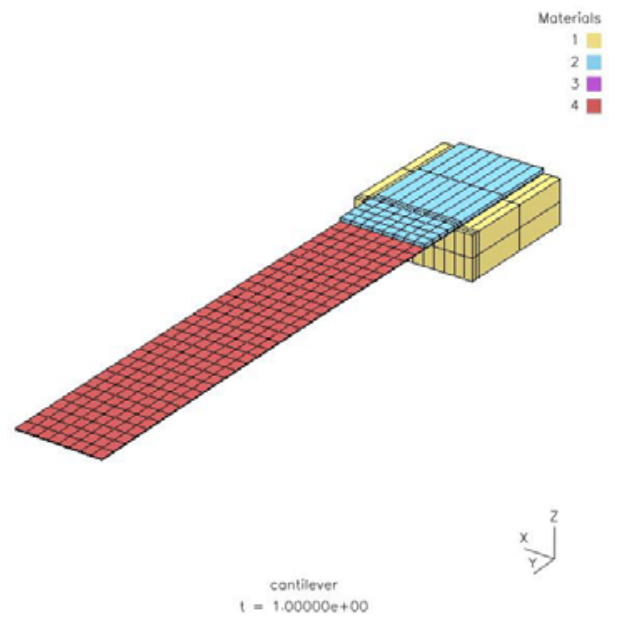

Fig. 26: Finite element mesh of a single cantilever. 


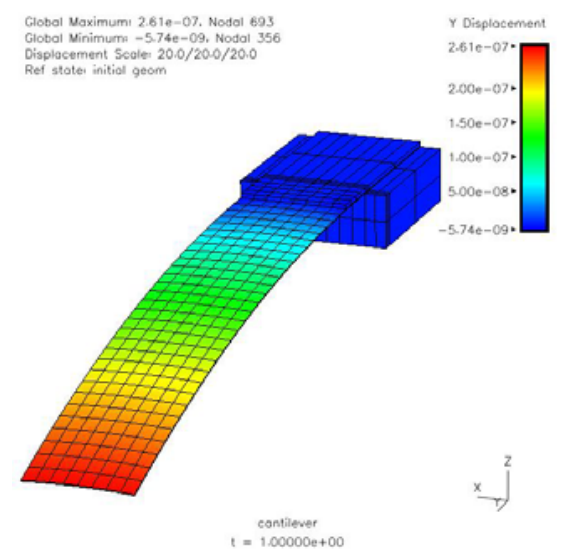

Fig. 27: Example of the deflection shape of a single cantilever under loading. Contours are of displacement in the normal (z) direction.

\subsection{Analytical relationships}

4.3.1 Deflection of a bi-metallic cantilever due to a change in temperature. From Roark and Young, 'Formulas for Stress and Strain,' Fifth Ed., Sect. 7.2[7],

Eq. 9

$$
\frac{\Delta z_{t i p}}{\Delta T}=3 \Delta \alpha L^{2} \frac{\delta_{A u}+\delta_{S i}}{\delta_{S i}^{2} k_{1}}
$$

where $\Delta \mathrm{z}_{\mathrm{tip}}$ is the normal (z-direction) deflection of the free end (tip) of the cantilever, $\Delta \mathrm{T}$ is the temperature difference, $\Delta \alpha$ is the difference in thermal expansion coefficients $\left(\alpha_{\mathrm{Si}}-\alpha_{\mathrm{Au}}\right), \mathrm{L}$ is the cantilever length, $\delta$ is the thickness of the $\mathrm{Si}$ or $\mathrm{Au}$, and $\mathrm{k}_{1}$ is

$$
k_{1}=4+6 \frac{\delta_{A u}}{\delta_{S i}}+4\left(\frac{\delta_{A u}}{\delta_{S i}}\right)^{2}+\frac{E_{A u}}{E_{S i}} \frac{\left(1-v_{S i}\right)}{\left(1-v_{A u}\right)}\left(\frac{\delta_{A u}}{\delta_{S i}}\right)^{3}+\frac{E_{S i}}{E_{A u}} \frac{\left(1-v_{A u}\right)}{\left(1-v_{S i}\right)}\left(\frac{\delta_{S i}}{\delta_{A u}}\right)
$$

where E is Young's modulus for Si or Au and $v$ is Poisson's ratio for Si or Au. Note the Poisson effect has been included in $\mathrm{k}_{1}$.

4.3.2 Deflection of a cantilever beam from a resultant surface stress. A modified version of Stoney's equation (applies free-free beam), which accounts for the fixed end boundary condition, comes from, Sader [8]. 
Eq. 10

$\Delta \sigma=\frac{1}{K}\left[\frac{1}{3}\left(\frac{\delta}{L}\right)^{2} \frac{E}{1-v} \Delta z\right]$

where

$K=X^{2}+2 v X\left\lfloor\frac{1}{\tau_{1}}+\frac{1}{\tau_{2}}\right\rfloor B+\left\lfloor\frac{1}{12}+2 v\left(\frac{1}{\tau^{2}{ }_{1}}+\frac{1}{\tau^{2}{ }_{2}}+\frac{1}{\tau_{1} \tau_{2}}\right)\right\rfloor B^{2}$

and

$\tau_{1,2}=2 \sqrt{3[5(1-v) \mp \sqrt{10(1-v)(2-3 v)}]}$

In these equations, $\Delta \sigma$ is the surface stress $\left(\mathrm{J} / \mathrm{m}^{\wedge} 2\right.$ or $\left.\mathrm{N} / \mathrm{m}\right), \delta$ is the thickness of the $\mathrm{Si}$ cantilever, $\mathrm{L}$ is the length of the cantilever, $\mathrm{E}$ is Young's modulus of $\mathrm{Si}, \mathrm{v}$ is Poisson's ratio for $\mathrm{Si}, \Delta \mathrm{z}$ is the normal (z-direction) deflection of the free end (tip) of the cantilever, $X=x / L=1$ is the normalized length, and $B=w / L$ is the normalized width of the cantilever. Note that $\Delta \sigma$, surface stress, is related to the surface stress energy per unit area for this cantilever geometry. (For details, see W. Haiss, [9])

$\mathrm{K}$ is the correction factor for Stoney's equation. For the case, $\mathrm{L}=500$ microns, $\mathrm{w}=100$ microns, and $\mathrm{t}=1$ micron, $\mathrm{K}=1.04$

4.3.3 Spring stiffness. A cantilever beam can be characterized by a 'spring stiffness' analogous to a one-degree-of-freedom mass/spring oscillator. The deflected shape corresponds to the first mode of vibration,

Eq. 11

$$
k=\frac{E w}{4}\left(\frac{\delta}{L}\right)^{3}
$$

where $\mathrm{E}$ is Young's modulus, $\mathrm{w}$ is the width, $\delta$ is the thickness, and $\mathrm{L}$ is the length. The moment of inertia of the cantilever with respect to the normal direction (often seen in literature) is

Eq. 12

$$
I=\frac{1}{12} w \delta^{3}
$$

4.3.4 Deflected shape of the cantilever. The normal displacement at any point along the cantilever is

Eq. 13

$$
z(x)=\frac{f}{2 E w \delta^{3}} x^{2}\left(x^{2}+6 L^{2}-4 L x\right)
$$


where $\mathrm{x}$ is the position along the cantilever ( $\mathrm{x}=0$ is the fixed end. $\mathrm{x}=\mathrm{L}$ is the free end). It is assumed that the cantilever is subject to a uniform loading, $\mathrm{f}$ (force/length).

The ratio of displacement at any point on the cantilever with respect to tip displacement is then

Eq. 14

$$
\frac{z(x)}{z_{\text {tip }}(L)}=\frac{x^{2}\left(x^{2}+6 L^{2}-4 L x\right)}{3 L^{4}}
$$

This relationship can be used when the measurement point is not located at the tip of the cantilever. For example, given a 500 micron cantilever, the displacement at a point 355 microns from the free end is $61.57 \%$ of the tip displacement.

\subsection{Finite element calculations vs. analytical relationships}

The following section uses the material properties values: Silicon: $\mathrm{E}=150 \mathrm{e} 9 \mathrm{~Pa}$, $v=0.2, \delta=1.0 \mathrm{e}-6 \mathrm{~m}, \mathrm{~L}=500 . \mathrm{e}-6 \mathrm{~m}, \alpha=4.2 \mathrm{e}-6 / \mathrm{C}$. Gold: $\mathrm{E}=80 \mathrm{e} 9 \mathrm{~Pa}, \mathrm{v}=0.42, \delta=$ 20e-9 m, L = 500.e-6 m, $\alpha=14.2 \mathrm{e}-6$ / C.

Consider tip deflection due to temperature difference: the analytical expression in Section 4.3.1 yields $\Delta \mathrm{z} / \Delta \mathrm{T}=103 \mathrm{~nm} / \mathrm{K}$. From the finite element model, $\Delta \mathrm{z} / \Delta \mathrm{T}=111$ $\mathrm{nm} / \mathrm{K}$. The analytical relationship is one dimensional. It does not take into account shortening in the axial direction. The finite element model does capture this effect. Figure 28 shows the corresponding amount of shortening in the axial direction for a 10 degree temperature increase. 


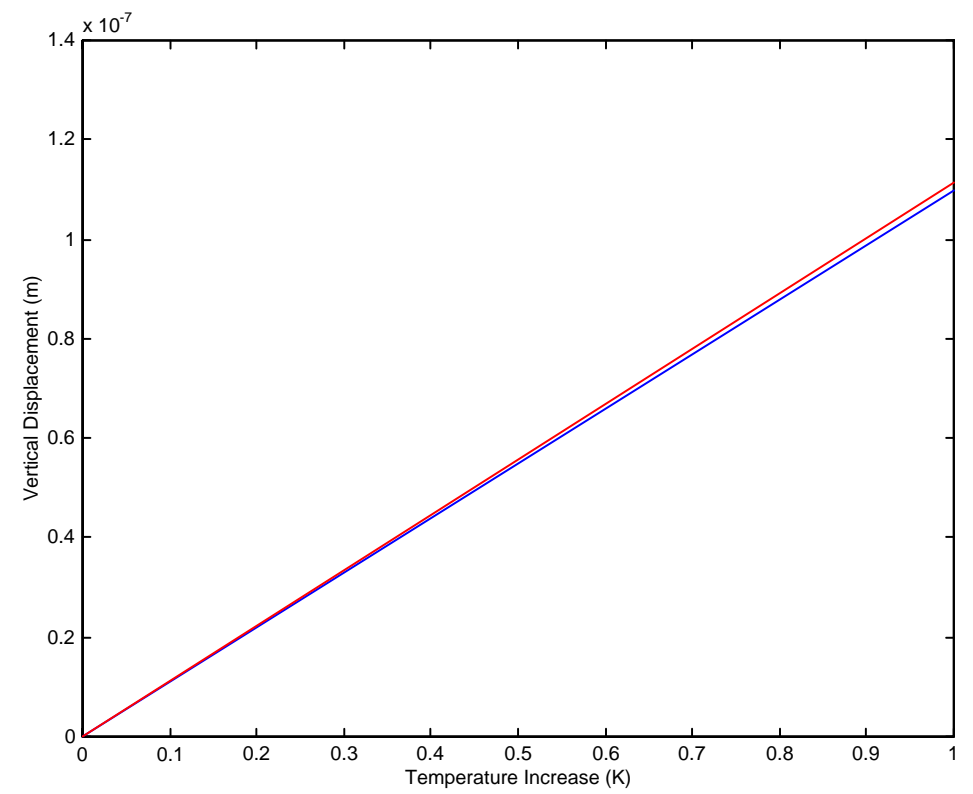

Fig. 28: Vertical displacement vs. temperature increase. Red: finite element model. Blue: analytical relation.

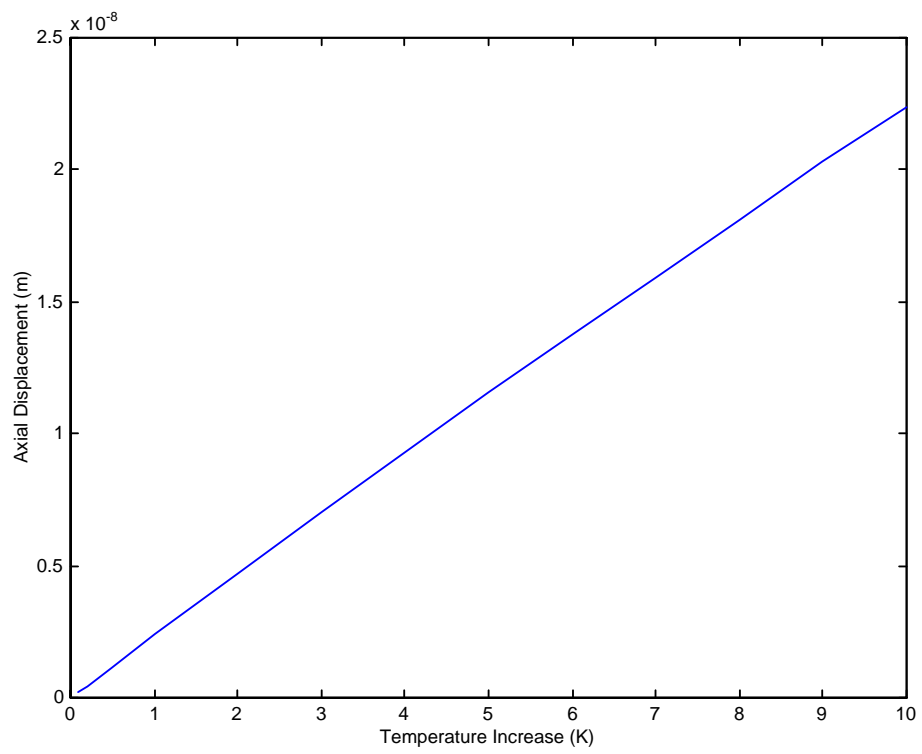

Fig. 29: Axial displacement vs. temperature increase from finite element model. The axial displacement is two orders of magnitude smaller than the vertical displacement. 


\subsection{Calibration of cantilevers using temperature-deflection-determined spring constant}

Material, geometric, and manufacturing (i.e., residual stress) variations in the cantilevers cause variations in mechanical response. Experimental measurements show that individual cantilevers respond to a given temperature change with varying displacement. We have attempted to characterize these variations through the spring constant (implemented for the purposes of our calculations in the Young's modulus) of each cantilever.

Experimentally, a known temperature increase in measured along with the corresponding cantilever displacement. Using the relationship in Section 4.3.1, a corresponding spring constant, $\mathrm{k}$, and a Young's modulus, E, can be calculated. The ideal value of $\mathrm{dz} / \mathrm{dT}$ is $104 \mathrm{~nm} / \mathrm{K}$ which corresponds $\mathrm{k}=30 \mathrm{nN} / \mathrm{m}$.

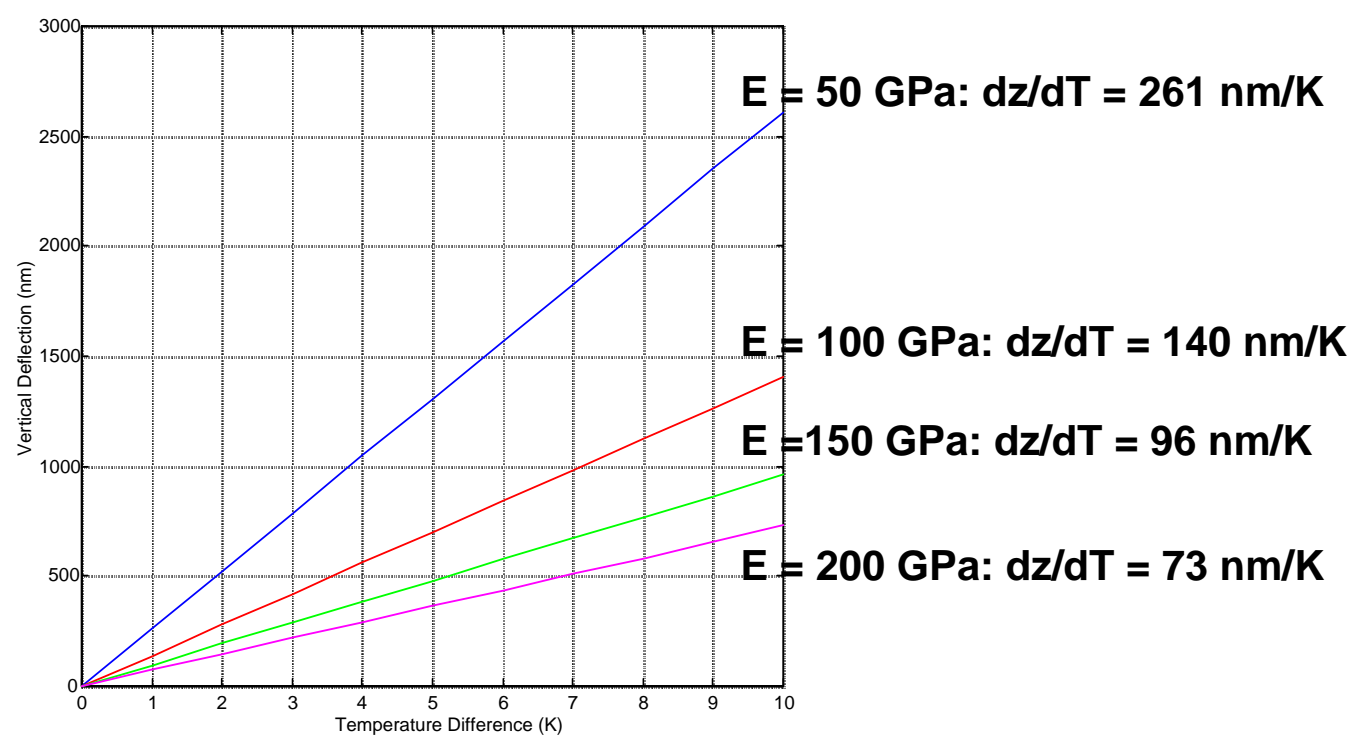

Fig. 30: Vertical displacement vs. temperature increase from four experimental measurements. For each dz / dT, an experimentally determined $\mathrm{E}$ is calculated.

An example of temperature vs. deflection data for eight cantilevers is shown in Fig. 31. A least-squares-fit line was used to estimate dz/dT for each lever. Then using the relationships in Sections 4.3.1 and 4.3.3, the corresponding spring constants were determined for each lever. The values ranged from $12.2 \mathrm{mN} / \mathrm{m}$ to $13.4 \mathrm{mN} / \mathrm{m}$. 


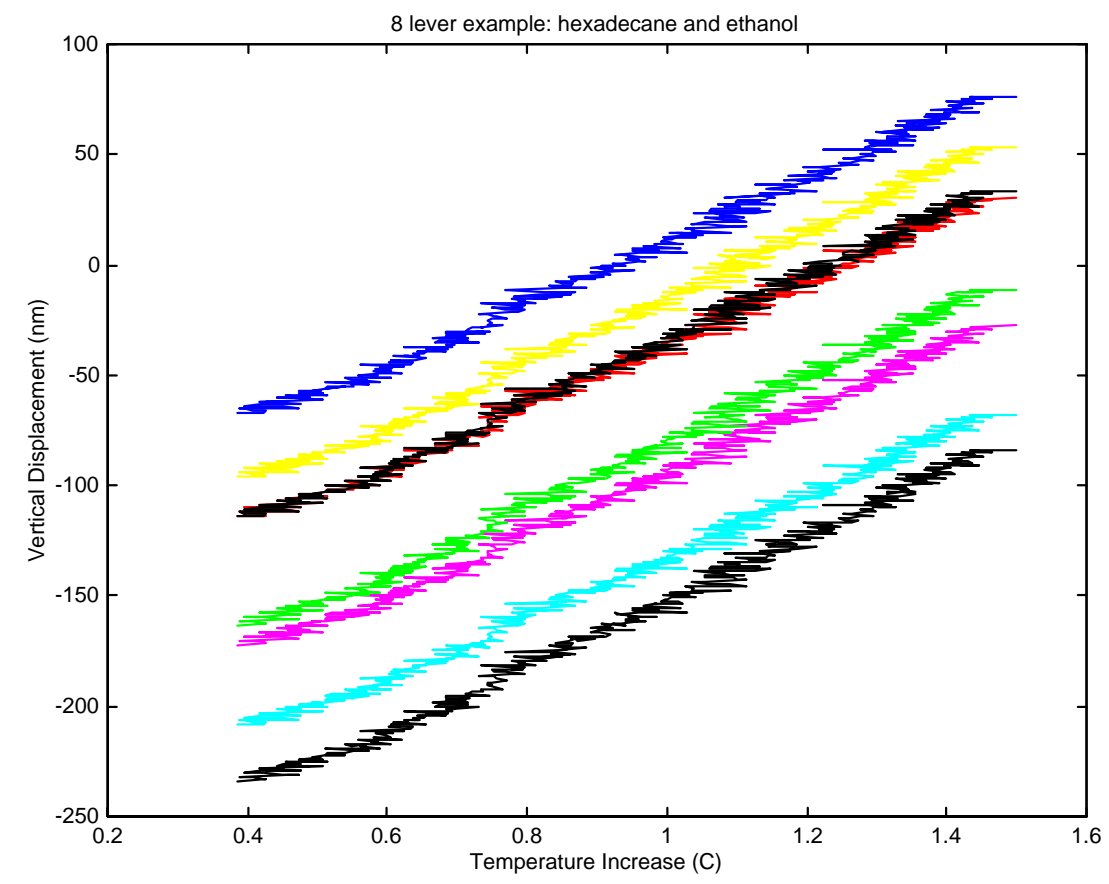

Fig. 31: Vertical displacement vs. temperature increase from a single temperature pulse. Displacement response of eight cantilevers. Spring stiffnesses varied from $12.2 \mathrm{mN} / \mathrm{m}$ to $13.4 \mathrm{mN} / \mathrm{m}$.

As an example, consider the data shown in Fig. 19. Here, we can fit to get the appropriate adsorption parameters. Furthermore, we also built in the cantilever response to thermal effects, or coefficient of thermal expansion effects. To estimate the contribution of CTE, we use Eq. 9 and find that $\Delta \mathrm{z} / \Delta \mathrm{T}$ approximately equals $98 \mathrm{~nm} / \mathrm{K}$.

The deflection, $\Delta \mathrm{z}$, is a function of material properties and temperature of the system. This estimate was based on literature values for material properties, and later in the project, the estimate was refined through temperature calibration experiments for each cantilever in the array. With all of these features built into the model based signal processing software, with fit parameters, we now have a capability to find a signal, with the appropriate features that our target species would produce, that is buried in non-signal or noise.

\section{Model-based signal processing of cantilever signals}

This section describes model-based processing, and how it is applied to achieve an enhanced signal for the cantilever array in a chemical detection problem.

\subsection{Model-based signal processing approach}

The model-based approach is "incorporating mathematical models of both physical phenomenology and the measurement process (including noise) into the 
processor to extract the desired information.” This approach provides a mechanism to incorporate knowledge of the underlying physics or dynamics in the form of mathematical process models (along with measurement system models and accompanying noise as well, as model uncertainties) directly into the resulting processor. In this way, the model-based processor enables the interpretation of results directly in terms of the problem physics. The model-based processor is actually a modeler's tool enabling the incorporation of any a priori information about the problem to extract the desired information.

The fidelity of the model incorporated into the processor determines the complexity of the model-based processor with the ultimate goal of increasing the inherent signal-to-noise ratio (SNR). These models can range from simple, implicit, non-physical representations of the measurement data such as the Fourier or wavelet transforms to parametric black-box models used for data prediction, to lumped mathematical representations characterized by ordinary differential equations, to distributed representations characterized by partial differential equation models to capture the underlying physics of the process under investigation. The dominating factor of which model is the most appropriate is usually determined by how severe the measurements are contaminated with noise and the underlying uncertainties. If the SNR of the measurements is high, then simple non-physical techniques can be used to extract the desired information; however, for low SNR measurements more and more of the physics and instrumentation must be incorporated for the extraction.

This paper is concerned with the novel development and application of modelbased signal processing to micro-machined cantilever sensor arrays. We will first functionally describe the generic approach in terms of the cantilever system and then we will develop the cantilever and associated mathematics more specifically eventually developing a first-cut design based on these models. The overall approach captures all possible models in terms of nonlinear differential equation models with the one described specifically an embellishment of the model-based approach.

We briefly describe the generic model-based approach to the cantilever array problem as depicted in Fig. 32. The movement of cantilevers is influenced by three major factors: chemical binding, temperature effects and fluidic forces. We will assume in this formulation that the temperature dynamics are relatively slow. Therefore, the changes in temperature can simply be modeled by a time-varying polynomial. Fluidic disturbances act more quickly, and can be described by the linear Stokes equations of motion. As a result, one can also solve the fluids problem separately. The resulting predicted stress can be superimposed over stresses due to temperature and chemical effects. Chemical reactions at the cantilever surface act over tens of seconds to many minutes, and represent the signal of interest. The entire process is contaminated with both measurement and process noise, which decreases the overall signal-to-noise ratio (SNR) and makes this invention so important. The poor signal levels can be enhanced by incorporating mathematical models of the underlying physics and measurements including the noise statistics into the processor to produce an enhanced signal (deflection) with a much higher SNR. 


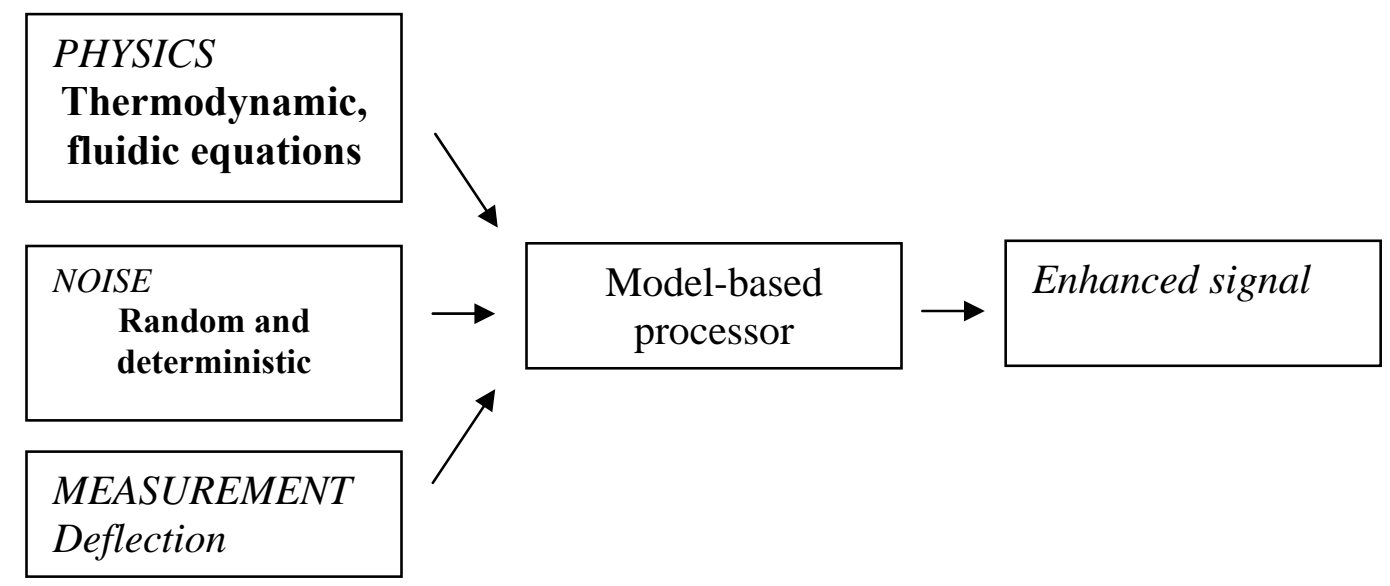

Fig. 32: Generic Model-Based Processing for Cantilever Sensor Arrays.

Mathematically, the time-dependent differential surface stress, $\sigma(t)$, on each cantilever is a function of the surface concentration given by $\Gamma(t)$, which is in turn parameterized by the maximum possible surface concentration, $\Gamma_{\max }$, and the initial target molecule concentration $\mathrm{c}_{0}$ and time-dependent target concentration $\mathrm{c}(\mathrm{t})$ in solution. The time rate of change of the surface concentration, $\Gamma(t)$, can be expressed as

$$
\dot{\Gamma}(t)=f_{\Gamma}\left[\Gamma(t), c_{0}, c(t), k_{a}, k_{d}, \Gamma_{\max }\right]+w(t),
$$

where $\mathrm{w}(\mathrm{t})$ is time-dependent process noise, assumed to be Gaussian, and all other terms are as defined above.

The change in a cantilever's' surface free energy, $\Delta \mathrm{G}(\mathrm{t})$, is related to the surface concentration and the target molecule concentration in solution by

Eq. 16

$$
\Delta G(t)=f_{\Delta G}\left[\Gamma(t), C(t), T(t), k_{a}, k_{d}\right]
$$

where $\mathrm{T}(t)$ is the time-dependent temperature. The differential surface stress is a function of the change in free energy and surface concentration by

Eq. 17

$$
\Delta \sigma(t)=f_{\Delta \sigma}[\Delta G(t), \Gamma(t)]
$$

With this underlying physical phenomenology we can now express the deflection at the $i^{\text {th }}$ cantilever as a function of both surface concentration and induced surface stress as

Eq. 18

$$
\Delta z_{i}(t)=f_{\Delta z}\left[\Delta \sigma_{i}(t), \Gamma_{i}(t)\right]
$$


Here we assume that the differential surface stress and as the surface concentration are variable. The actual measurement is somewhat more complicated due to instrumentation noise $v(t)$ as well as disturbances $\delta(t)$ such as systematic errors, temperature drifts and just trends. Therefore, we define the cantilever array deflection measurement at the $i^{\text {th }}$ lever as

Eq. 19

$$
d_{i}(t)=f_{d_{i}}\left(\alpha_{i}, \Delta z_{i}, \delta_{i}\right)+v_{i}(t)
$$

with $\alpha_{i}$ a gain or attenuation assumed constant. In this characterization, we also assume that the additive noise processes are zero-mean and Gaussian. This representation can be further parameterized in terms of an approximate Gauss-Markov model with nonlinear dynamics and algebraic equations. We will discuss this subsequently. This completes the generic description of the system cast into the model-based framework, next we define some more specific representations based on the underlying phenomenology. 


\subsection{Model-based processor design for cantilever arrays}

In this section we develop the model-based approach first for the generic model sets and then for the specific embellishment discussed previously. We start the section with the development of an approximate Gauss-Markov model, which can be used to capture the cantilever signal enhancement problem and then specifically apply it to the micro-cantilever array sensor system.

If we have nonlinear dynamics (differential equations) describing the system under investigation, then an approximate representation of the deterministic process and associated measurement is easily captured in state-space form. State-space is simply converting an $n^{\text {th }}$-order set of coupled differential equations into an equivalent set of $n$ first order differential equations. With this accomplished, we obtain the general nonlinear vector functional relations defined by the process and measurement models,

$$
\dot{x}(t)=a[x, u ; \theta] \quad \text { [state }]
$$

Eq. 20

$$
y(t)=c[x, u ; \theta] \quad \text { [measurement] }
$$

where $x$ is the $N_{x}$-dimensional state vector; $y$ is the $N_{y}$-dimensional measurement. If these processes are contaminated by additive zero-mean, Gaussian noise processes then the approximate Gauss-Markov model evolves as

$$
\dot{x}(t)=a[x, u ; \theta]+\mathrm{w}(\mathrm{t}) \quad \text { [process (state) }]
$$

Eq. 21

$$
y(t)=c[x, u ; \theta]+\mathrm{v}(\mathrm{t}) \quad \text { [measurement] }
$$

Here $a[\cdot], \mathrm{c}[\cdot]$ are the $N_{x}$-dimensional vector process function and $N_{y}$-dimensional measurement functions, respectively, for the process noise given by $w \sim N\left(0, R_{w w}\right)$ and the corresponding measurement noise as $v \sim N\left(0, R_{v v}\right) . N(\cdot, \cdot)$ is the Gaussian distribution specified by mean and covariance. With this representation in mind, we can now define the cantilever signal enhancement problem as:

GIVEN a set of noisy displacement measurements, $\{y(t)\}$ with known inputs, $\{u(t)\}$ and parameters, $\{\theta\}$ specified by the approximate Gauss-Markov model of Eq. 21, FIND the best (minimum error variance) estimate of the displacement and surface concentrations, $\hat{y}(t), \hat{x}(t)$, respectively. 
The solution to this problem can be derived in a wide variety of approaches. Here, we will use the common Bayesian approach[10]. We summarize the algorithm as:

\section{MODEL-BASED PROCESSOR ALGORITHM}

$$
\dot{\hat{x}}(t \mid t-1)=a[\hat{x}, u ; \theta]
$$

Eq. $22 \hat{y}(t \mid t-1)=c[\hat{x}, u ; \theta]$

$$
\begin{aligned}
& \varepsilon(t)=y(t)-\hat{y}(t \mid t-1) \\
& \hat{x}(t \mid t)=\hat{x}(t \mid t-1)+K(t) \varepsilon(t)
\end{aligned}
$$

[State Prediction]

[Measurement Prediction]

[Innovation or Residual]

[Correction]

where $K(t)$ is the gain of the processor, which must be calculated from the underlying process statistics (see Candy, Signal Processing: The Model Based Approach (1986)[10]) for details.

For the micro-cantilever array problem, we must convert the physical relations, that is, Eqs. 2-4 into the state-space form above. We chose to solve the differential equation and incorporate the resulting relations into the measurement model. For the state we modeled the free energy as a piecewise constant function, converted it to discrete-time using the first difference approximation and excite it with zero-mean, white Gaussian (process) noise creating a random walk model for this parameter. Therefore, we start with defining the state vector as, $x:=\Delta G$ and the measurement, $y:=d$, then we obtain the following relations. From Eq. 22, the surface concentration relation becomes

Eq. 23

$$
x(t)=x(t-1) \rightarrow a[x ; \theta]
$$

[Process Model]

Now the measurement model is tricky. We must first solve for the physical variables to obtain the generic form of Eq. 21; therefore, we have from Eqs. 2-4 that
Eq. 24

$$
y_{\ell}(t)=d_{\ell}(t)+\tau(t) \rightarrow c[x, u ; \theta]
$$
[Measurement Model]

The dynamic surface concentration, $\Gamma(\mathrm{t})$ for adsorption-desorption and pure desorption processes that were used have the following form: 
Eq. 25

$\Gamma(t)=\left\{\begin{array}{cc}0 & t<t_{\text {ON }} \\ \beta\left(1-e^{\left(-k_{a} C(t)+k_{d}\right)\left(t-t_{O N}\right)}\right) & \text { for } \quad t_{\text {ON }} \leq t \leq t_{\text {OFF }} \\ \sqrt{\frac{1}{2 k_{d}\left(t-t_{\text {OFF }}\right)}} & t>t_{\text {OFF }}\end{array}\right.$

Here $\beta=\left(\frac{c(t)}{c(t)+K_{E Q}}\right) ; \quad K_{E Q}=k_{a} / k_{d}$, and ton denotes when the signal is introduced and $t_{\text {Off }}$ denotes when the signal is turned off.

As described above, the differential surface stress on the cantilever is a function of the surface concentration and free energy

Eq. 26

$$
\Delta \sigma(t)=\Gamma(t) \Delta G(t) M^{-1}=\Gamma(t) x(t)
$$

Therefore the deflection of the $\ell^{\text {th }}$-lever is weighted by the Stoney equation with a specific modulus of the individual lever, that is,

Eq. 27

$$
d_{\ell}(t)=\alpha_{\ell} \Delta \sigma(t) \quad \text { for } \quad \alpha_{\ell}:=\frac{3 L^{2}}{E_{\ell} \delta}(1-v)
$$

where $\alpha_{\ell}$ is the $\ell^{\text {th }}$ Stoney equation with cantilever modulus, $E_{\ell}$, with lever length and thickness, $L, \delta$, respectively and $v$ is Poisson's ratio for silicon. Thus, we obtain the measurement equation at the $\ell^{\text {th }}$-cantilever as

Eq. 28

$$
y_{\ell}(t)=\zeta_{\ell}(t) x(t)+\tau(t) \text { for } \zeta_{\ell}(t):=\alpha_{\ell} \Gamma(t)
$$

and $\tau(t)$ is the known temperature profile (low-pass filtered). Finally, assuming that both noise sources are Gaussian random processes (as before), then the result is a time-varying Gauss-Markov (not approximate due to linearity) multi-channel cantilever model defined by 


$$
x(t)=x(t-1)+w(t-1)
$$

Eq. 29

$$
y_{\ell}(t)=\zeta_{\ell}(t) x(t)+\tau(t)+v_{\ell}(t)
$$

for $w \sim N\left(0, R_{w w}\right) ; v \sim N\left(0, R_{v v}\right)$. Then as before, we can develop the model-based processor based on this Gauss-Markov model. First, we define the signal enhancement problem in terms of the cantilever models as:

GIVEN a set of noisy $N_{y}$-vector displacement measurements, $\{\mathbf{y}(t)\}$ with known $N_{u}$-vector inputs, $\{\mathbf{u}(t)\}$ and parameters, $\left\{\theta_{k}\right\}$ specified by the Gauss-Markov model of Eq. 27, FIND the best (minimum error variance) estimate of the displacement and $N_{x}$-vector surface concentrations, $\hat{\mathbf{y}}(t \mid t-1), \hat{\mathbf{x}}(t \mid t)$, respectively.

The model-based algorithm to solve this problem using the specified models is:

Eq. 30

\begin{tabular}{|lc|}
\hline \multicolumn{2}{|c|}{ CANTILEVER ARRAY MODEL-BASED PROCESSOR ALGORITHM } \\
$\dot{\hat{\mathbf{x}}}(t \mid t-1)=\hat{\mathbf{x}}(t-1 \mid t-1)$ & [Surface Concentration Prediction] \\
$\hat{\mathbf{y}}_{\ell}(t \mid t-1)=\zeta_{\ell}(t) \hat{\mathbf{x}}(t \mid t-1)+\hat{\tau}(t)$ & [Displacement Prediction] \\
$\boldsymbol{\varepsilon}_{\ell}(t)=\mathbf{y}_{\ell}(t)-\hat{\mathbf{y}}_{\ell}(t \mid t-1)$ & [Innovation or Residual] \\
$\hat{\mathbf{x}}(t \mid t)=\hat{\mathbf{x}}(t \mid t-1)+\mathbf{k}(t) \boldsymbol{\varepsilon}(t)$ & [Surface Concentration Correction] \\
\end{tabular}

This completes the development of the MBP algorithm for Cantilever Sensor Arrays. Note that once this framework is developed, it is quite easy to define other problems of high interest (e.g. detection problems [11]).

\subsection{Model-based processor performance evaluation}

In this section we discuss the performance of the model-based processor (MBP) for signal enhancement of an $L$-element cantilever sensor array. The basic approach we 
take for MBP performance evaluation is illustrated in Fig. 33. After obtaining the average parameters by performing the parameter estimation, a Gauss-Markov simulation was designed to generate synthesized cantilever deflection measurements using the model discussed in the previous section. Once synthesized at a particular signal-to-noise ratio (SNR), the processors were applied to the data and their performance analyzed based on the "truth" deflections generated by a noise-free simulation. Performance metrics are applied to evaluate and compare performance. We discuss the various steps in this procedure.

\section{Parameter Estimation}

The basic approach we use is to first "parameterize" the cantilever array model by performing parameter estimation ${ }^{\nabla}$ on the raw deflection measurements to extract the critical absorption, desorption and maximum concentration, that is, $\theta_{\ell}=\left\{k_{a}(\ell), k_{d}(\ell), \Delta G(\ell)\right\} ; \quad \ell=1, \cdots, L$. The parameter estimator we employed was a nonlinear least-squares criterion using the Nelder-Meade polytope search algorithm [12]. This algorithm is based on minimizing

Eq. 31

$$
\min _{\theta_{\ell}} J(\theta)=\sum_{t=1}^{N_{t}} \varepsilon_{\ell}^{2}(t ; \theta) \text { for } \varepsilon(t ; \theta):=y_{\ell}(t)-\hat{y}_{\ell}(t ; \theta) \text {, }
$$

where the estimated or filtered cantilever measurement at the $\ell^{\text {th }}$-lever is given by

Eq. 32

$$
\hat{y}_{\ell}(t ; \theta)=\hat{d}_{\ell}(t ; \theta)+\tau(t) \text {. }
$$

Once these parameters are extracted from the data, they are averaged to give, $\overline{\boldsymbol{\theta}}=\left\{\overline{k_{a}}, \overline{k_{d}}, \overline{\gamma_{\max }}\right\}$. These are the parameters that are used in the Gauss-Markov simulation model.

We ran this parameter estimator on raw experimental deflection data and estimated the parameters for each lever. The results are shown in Fig. 34 where we see the "fitted" deflection responses compared to the measured. It is clear that the extracted parameters reasonably predict the filtered cantilever response of Eq. 31. Next we investigate the development of the simulator.

\footnotetext{
${ }^{\nabla}$ We used a nonlinear least-squares algorithm in MATLAB to estimate the parameters.
} 


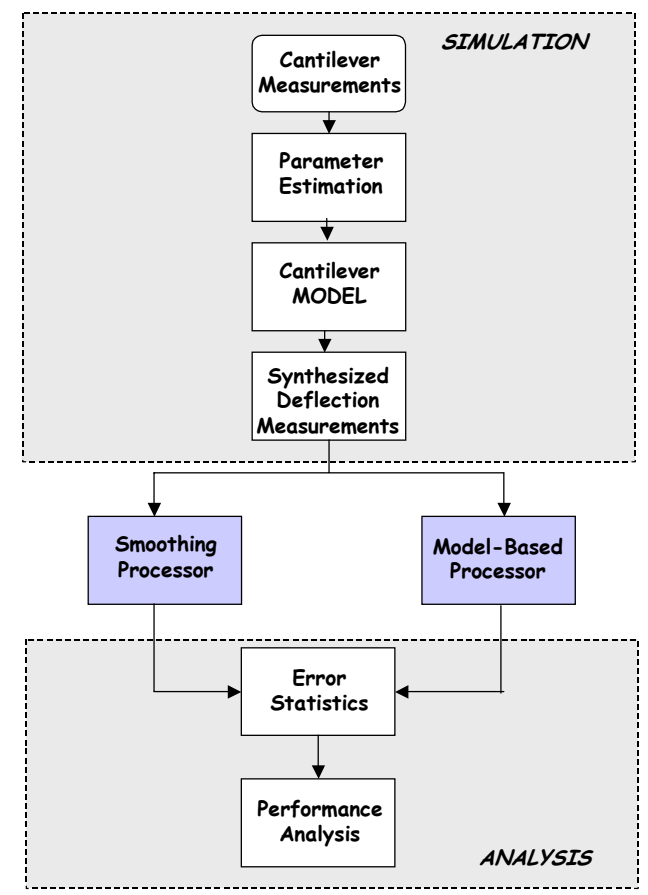

Fig. 33: Model-Based Processor Performance Evaluation: Simulation, Processing and Analysis.
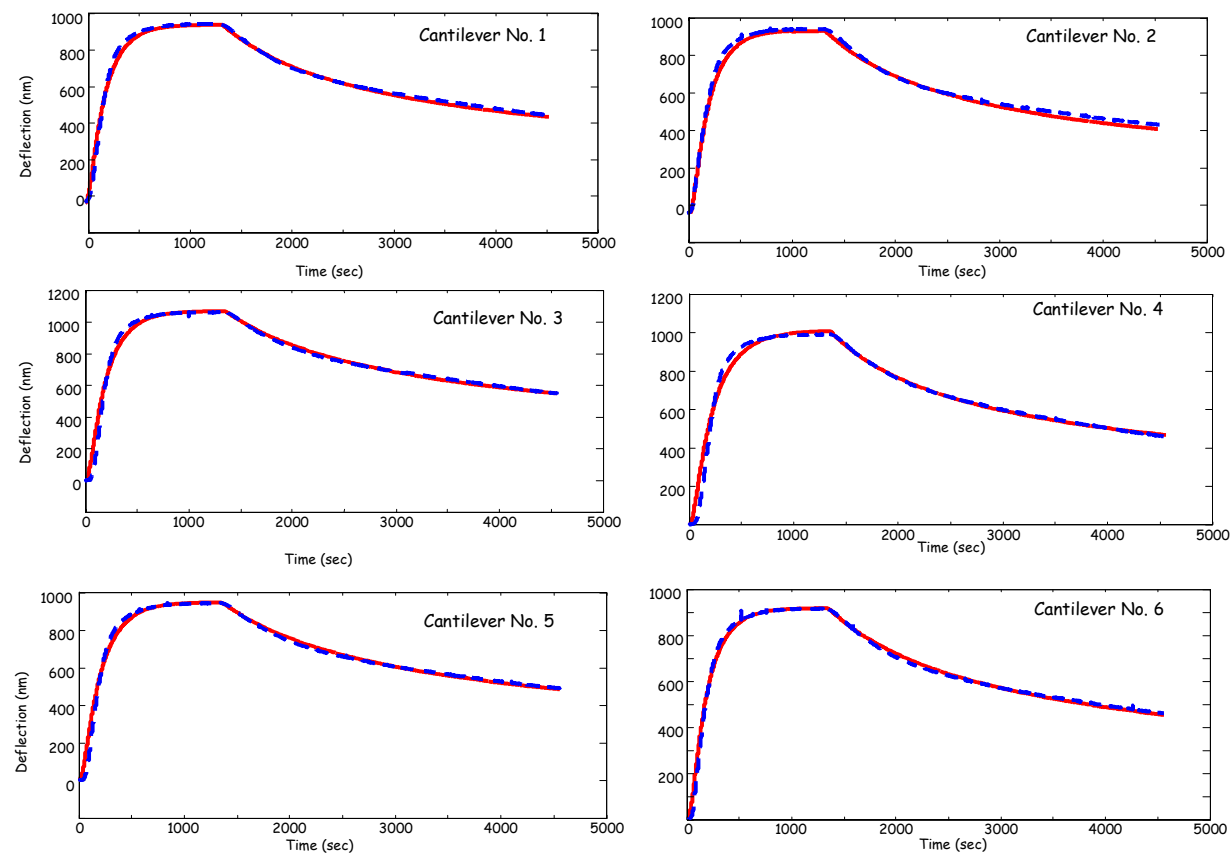

Fig. 34: Parameter Estimator Results: Predicted response (solid line) compared to raw deflection measurements (dotted line). Note: $C_{0}$ is 1.4E-2 M, mercaptoethanol in water, for this experiment. 
Table 2. Cantilever Parameter Estimation

for 1.4 E-2 M Mercaptoethanol in Water

\begin{tabular}{|c|c|c|c|}
\hline Cantilever & $\mathrm{k}_{\mathrm{a}}\left(\mathrm{M}^{-1} \mathrm{sec}^{-1}\right)$ & $\mathrm{k}_{\mathrm{d}}\left(10^{-4}\right)\left(\mathrm{sec}^{-1}\right)$ & $\Gamma_{\max }\left(10^{15}\right)$ \\
\hline No. 1 & 0.333555 & 5.1484 & 1.27565 \\
\hline No. 2 & 0.329629 & 5.5826 & 1.32083 \\
\hline No. 3 & 0.319429 & 4.3686 & 1.35710 \\
\hline No. 4 & 0.329063 & 4.7588 & 1.26563 \\
\hline No.5 & 0.310646 & 4.4155 & 1.21784 \\
\hline No. 6 & 0.342959 & 4.7652 & 1.16820 \\
\hline & & & \\
\hline AVERAGE & 0.32755 & 4.8398 & 1.26751 \\
\hline & & & \\
\hline
\end{tabular}

For comparison: $\mathrm{k}_{\mathrm{a}}$ is $1.3 \mathrm{E}-2 \mathrm{M}^{-1} \mathrm{sec}^{-1}$ for $1 \mathrm{mM} \mathrm{CH}_{3}\left(\mathrm{CH}_{2}\right)_{15} \mathrm{~S} / \mathrm{Au}$ in ethanol [13]. $\Delta \mathrm{G}=$ -RT Ln $\left(\mathrm{k}_{\mathrm{a}} / \mathrm{k}_{\mathrm{d}}\right)=-24 \mathrm{E} 3 \mathrm{~J}$ for this system; for 718 base pair thiolated DNA, $\Delta \mathrm{G}=-34.6 \mathrm{E} 3$ $\mathrm{J}$ [4]. $\Gamma_{\max }=1.3 \mathrm{E} 13$ for 20-mer DNA [14].

\section{Gauss-Markov Model Simulation}

For our problem we chose to use $\Delta G(t)$ as an unknown but constant parameter $(\Delta \dot{G}(t)=0$ ) and the nonlinear deflection and known temperature measurement, that is, defining

Eq. 33

$$
\Delta G(t)=\Delta G(t-1)+w(t-1)
$$

with cantilever array measurement

Eq. 34

$$
y_{\ell}(t)=d_{\ell}(t)+\tau(t)+v_{\ell}(t)
$$

where $\Delta G(t)$ is the free energy at the surface, $d_{\ell}$ is the displacement of the $\ell^{\text {th }}$-lever, $\tau$ is the temperature of the fluid medium and $w, v_{\ell}$ are the additive, zero-mean, Gaussian noise processes with covariances, $R_{w w}$ and $R_{v v} \in \mathbf{R}^{L \times L}$ with diagonals, $\sigma_{v}^{2}(\ell) ; \ell=1, \cdots, L$. We assume that the measurement uncertainty is uncorrelated producing the diagonal matrix. Each of the cantilevers has a different modulus associated with its structure creating a set of Stoney equation parameters, $\alpha \rightarrow \alpha_{\ell}$, one representing each of the individual lever dynamics. A typical set of cantilever simulation data is shown in Fig. 35 where we used a $-20 d B$ SNR defined by 
Eq. 35

$$
S N R_{\ell}=\frac{\sigma_{D \text { true }}^{2}(\ell)}{\sigma_{v}^{2}(\ell)} ; \quad \ell=1, \cdots, L
$$

where $D_{\text {true }}$ is the "true" deflection available from the Gauss-Markov simulation and $\sigma_{D \text { true }}^{2}$ is its variance. Once the noisy deflection measurements are synthesized, then the processors are applied to extract the "true" deflections. We chose to evaluate two methods: (i) smoothing processor; and (2) model-based processor. The smoothing processor is simply a running window average that is equivalent to a low-pass filtering operation. This is an example of a typical approach taken by scientists in this field. The MBP is the "optimal” (approximately) solution to this problem. Note that we used the third party toolbox, SSPACK_PC, a commercial model-based signal processing package[15].
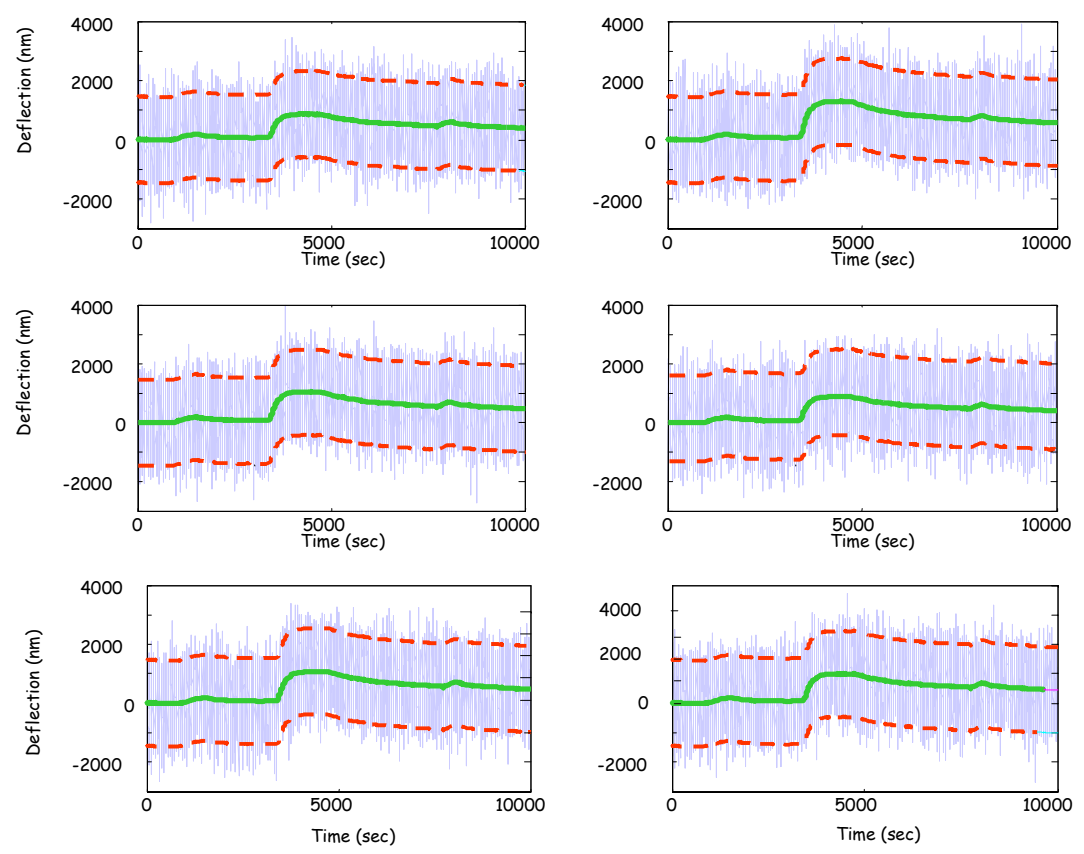

Fig. 35: Noisy Cantilever Deflection Measurement Gauss Markov Simulation (-20 dB SNR).

\section{Model-Based Processor Design}

The MBP was developed using the cantilever measurement model of Eq. 34 with the average parameter estimates of Table 2 . In this section we discuss the application of the MBP to simulated cantilever array measurement data at a variety of SNRs. We first show the results of the MBP design for the $-20 d B$ SNR case and then summarize the results at a variety of SNRs to evaluate its overall performance. Note that even though the data were simulated using the recorded temperature, it was removed (subtracted) from the final estimates in calculating the corresponding error statistics. The MBP design discussed is based not only on the average parameter estimates (see Table 3), but also 
using a smoothed temperature data estimate, $\hat{\tau}(t)$, to be more realistic in performance evaluation. We expect this processor to provide an outstanding performance, once tuned [10]. In fact, the results of applying to be $-20 \mathrm{~dB}$ data indicating an approximate optimal performance, since the underlying prediction errors or innovations associated with each lever are statistically zero-mean and white as shown in Fig. 36 and Table 3. Table 3 also shows the aggregated weighted-sum squared residual (WSSR) statistic indicating optimal performance. The results of processing the $-20 \mathrm{~dB}$ SNR deflection data are shown in Fig. 37 for each lever. The results are shown by comparing the "true" (synthesized) deflection compared to the smoothed and MBP estimates. It is clear from the figure that the MBP performs extremely well.
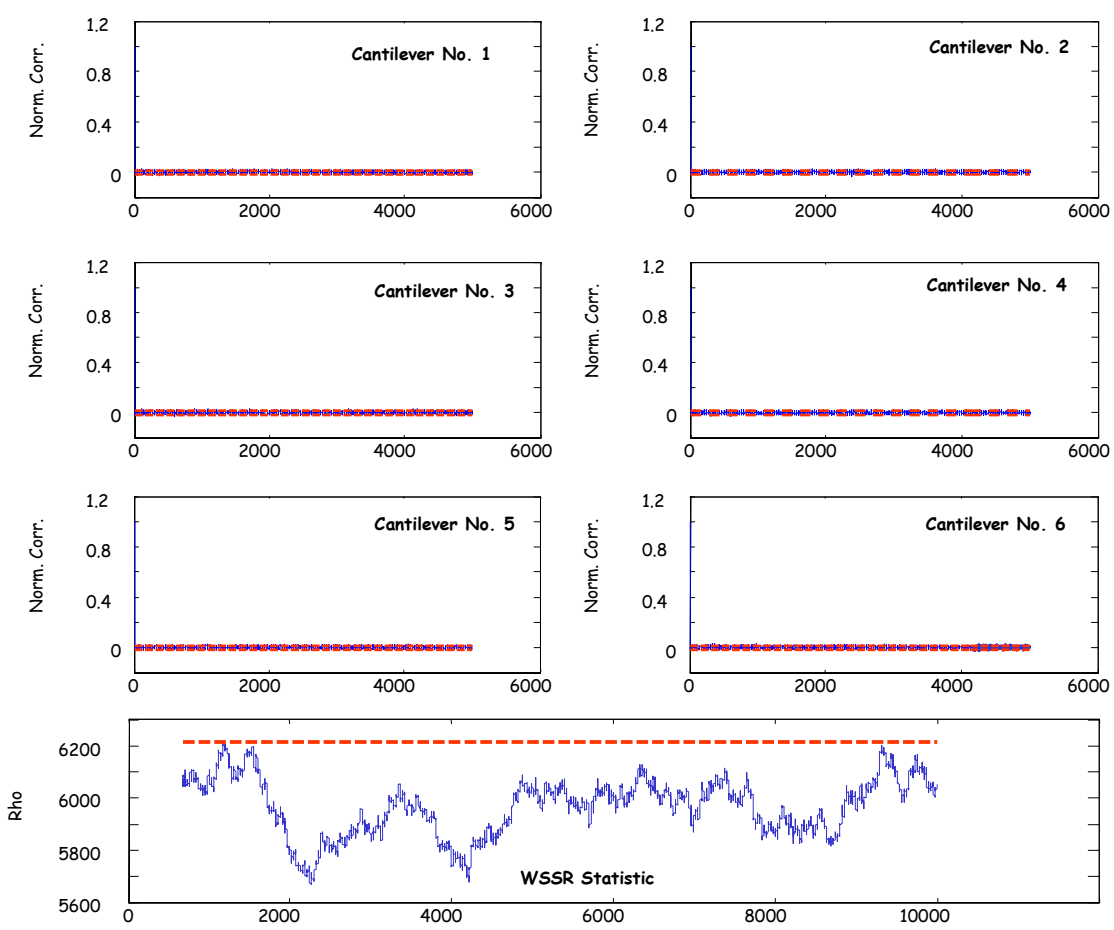

Fig. 36: Optimality Tests: Cantilever Array Zero-Mean/Whiteness and WSSR Statistic. 
Table 3. MBP Optimality Zero-Mean/Whiteness Test Results

\begin{tabular}{|c|c|c|c|c|}
\hline Cantilever & Mean & Bound & Whiteness (\% out) & Pass/Fail \\
\hline No. 1 & 7.64 & 16.7 & 4.25 & $\mathrm{P}$ \\
\hline No. 2 & 7.81 & 16.6 & 4.48 & $\mathrm{P}$ \\
\hline No. 3 & 11.6 & 16.9 & 4.39 & $\mathrm{P}$ \\
\hline No. 4 & 8.98 & 16.5 & 3.59 & $\mathrm{P}$ \\
\hline No. 5 & 1.93 & 16.8 & 4.51 & $\mathrm{P}$ \\
\hline No. 6 & 4.16 & 16.6 & 4.34 & $\mathrm{P}$ \\
\hline & & & & \\
\hline WSSR & & & < Threshold & $\mathrm{P}$ \\
\hline
\end{tabular}
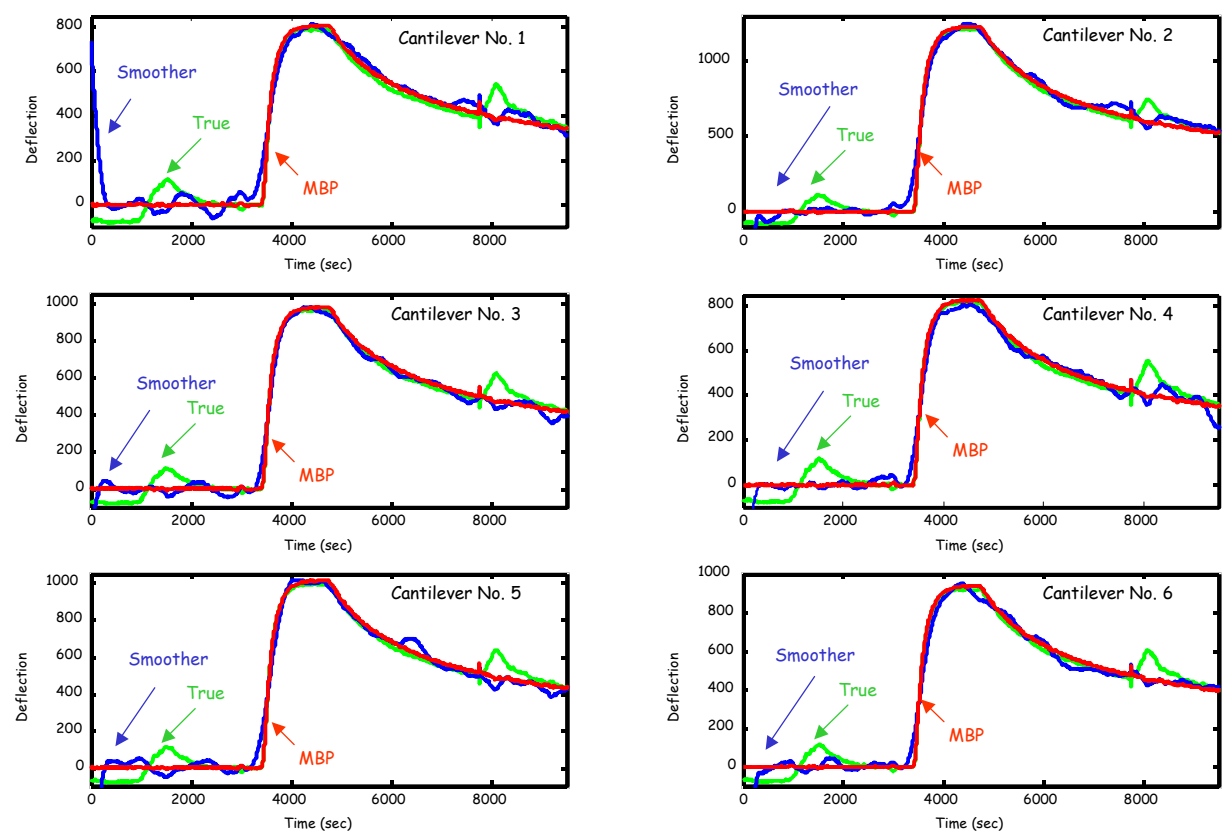

Fig. 37: MBP and Smoother Enhancement of the Noisy (-20 dB SNR) Synthesized Deflection Measurements: True Deflection (with temperature data), Smoothed and MBP Estimates. 
Next we investigate the overall performance of both the standard smoother/averager and the MBP on synthesized data sets. In order to quantitatively evaluate the performance of the processors individually we calculate the residual deflection errors defined by

Eq. 36

$$
\tilde{d}_{\ell}(t):=d_{\text {true }_{\ell}}(t)-\hat{d}_{\ell}(t) \text {, }
$$

where $d_{\text {true }}$ is the true (noise free) deflection at the $\ell^{\text {th }}$-lever and $\hat{d}_{\ell}$ is the filtered or estimated deflections as shown for the $-20 d B$ case in Fig. 37. Once the error is estimated at each lever then its associated statistics mean and variance can be calculated and used for further analysis. We define the processor output gain as the final metric given by

Eq. 37

$$
\operatorname{SNR}_{\text {out }}(\ell):=\frac{\sigma_{d_{\text {true }}}^{2}(\ell)}{\sigma_{\tilde{d}}^{2}(\ell)} ; \quad \ell=1, \cdots, \mathrm{L} .
$$

This ratio represents the enhancement provided by each processor. The smaller the residual error variance $\left(\sigma_{\tilde{d}}^{2}\right)$, the higher the $S N R_{\text {out }}$, providing a reasonable metric. Averaging these statistics over the cantilever array gives a "feel” for overall processing gain.

We performed a set of simulations at $0,-20,-40 d B$ SNR $_{\text {in }}$ with the typical run outputs shown in the previous figures for the $-20 d B$ case. The results for each run are summarized in Table 4. Here we see that output SNR produced by both processors are respectable with a significant gain in enhancement; however, it is clear that the MBP consistently demonstrates superior performance with an overall average enhancement of $80 \mathrm{~dB}$ and enhancement gain over the Smoother of 38, 43 and $60 \mathrm{~dB}$, respectively. The MBP is insensitive at these SNRs to the measurement noise variance changes yielding identical performance at each level. By contrast, the Smoother performance clearly deteriorates as the input SNR decreases. The MBP performance will also deteriorate with decreases in input SNR, but not at realistic experimental levels. This demonstrates outstanding performance for multi-channel cantilever arrays on these simulated data sets. Next we apply the process to the measured array data. 
Table 4. MBP/Smoother Performance Analysis

\begin{tabular}{|c|c|c|c|c|c|c|}
\hline $\mathrm{SNR}_{\text {in }}(\mathrm{dB})$ & 0 & 0 & -20 & -20 & -40 & -40 \\
\hline Cantilever & $\mathrm{SNR}_{0}(\mathrm{Smt})$ & $\mathrm{SNR}_{0}(\mathrm{MBP})$ & $\mathrm{SNR}_{0}(\mathrm{Smt})$ & $\mathrm{SNR}_{0}(\mathrm{MBP})$ & $\mathrm{SNR}_{0}(\mathrm{Smt})$ & $\mathrm{SNR}_{0}(\mathrm{MBP})$ \\
\hline & & & & & & \\
\hline No. 1 & 44.0083 & 79.9439 & 36.5903 & 79.9439 & 17.6070 & 79.9440 \\
\hline No. 2 & 46.0711 & 87.2620 & 40.2018 & 87.2620 & 26.3940 & 87.2620 \\
\hline No. 3 & 44.9283 & 83.3775 & 44.5746 & 83.3775 & 23.5760 & 83.3770 \\
\hline No. 4 & 45.2004 & 80.3783 & 37.9417 & 80.3783 & 17.3240 & 80.3780 \\
\hline No. 5 & 44.8704 & 83.8749 & 39.8227 & 83.8749 & 22.9630 & 83.8750 \\
\hline No.6 & 45.9690 & 82.4926 & 40.0839 & 82.4926 & 24.0330 & 82.4930 \\
\hline & & & & & & \\
\hline AVG & 45.1746 & 82.8882 & 39.8691 & 82.8882 & 21.983 & 82.888 \\
\hline AvgGain & & +37.7136 & & +43.0191 & & +60.905 \\
\hline
\end{tabular}

\subsection{Model-based processor application to multichannel cantilever data}

In this section we developed MBP for two cases: (1) average deflection data and an averaged cantilever model; and (2) multi-channel deflection data. First we averaged the 6 cantilever deflection data to obtain the average deflection response over the entire array and then designed the MBP obtaining the parameter estimates first and then applying the processor with those parameters. Next we developed the multi-channel processor using the same approach by estimating the individual cantilever parameters, taking their average and calculating the appropriate moduli for the multi-channel model. In both cases we performed simulations first to develop the optimal MBP.

The average cantilever model was developed by performing the model-based parameter estimation (see Fig. 9c) obtaining the adsorption, desorption and free energy and then developing the corresponding Gauss-Markov simulation model and corresponding MBP. The raw and simulated data $(0 d B)$ are shown in Fig. 38a and 38b. It is clear that the measurement noise severely distorts the desired deflection signal. The enhanced MBP output (deflection) enhancement is shown in Fig. 38c along with the corresponding optimality tests in 38d where we see that the performance of the processor is indeed optimal since the corresponding innovations are zero-mean and white. Next we applied the MBP to the actual deflection and temperature profile data shown in Fig. 39a and 39b after "tuning" the noise covariance parameters $\left(R_{v v}\right)$ with the results shown in Fig. 39d. Here we see that the MBP is capable of tracking the averaged cantilever deflection data quite well; however, the performance is suboptimal, since the innovation, although quite small, are not white. Generally, the MBP performance for this data is quite good. 

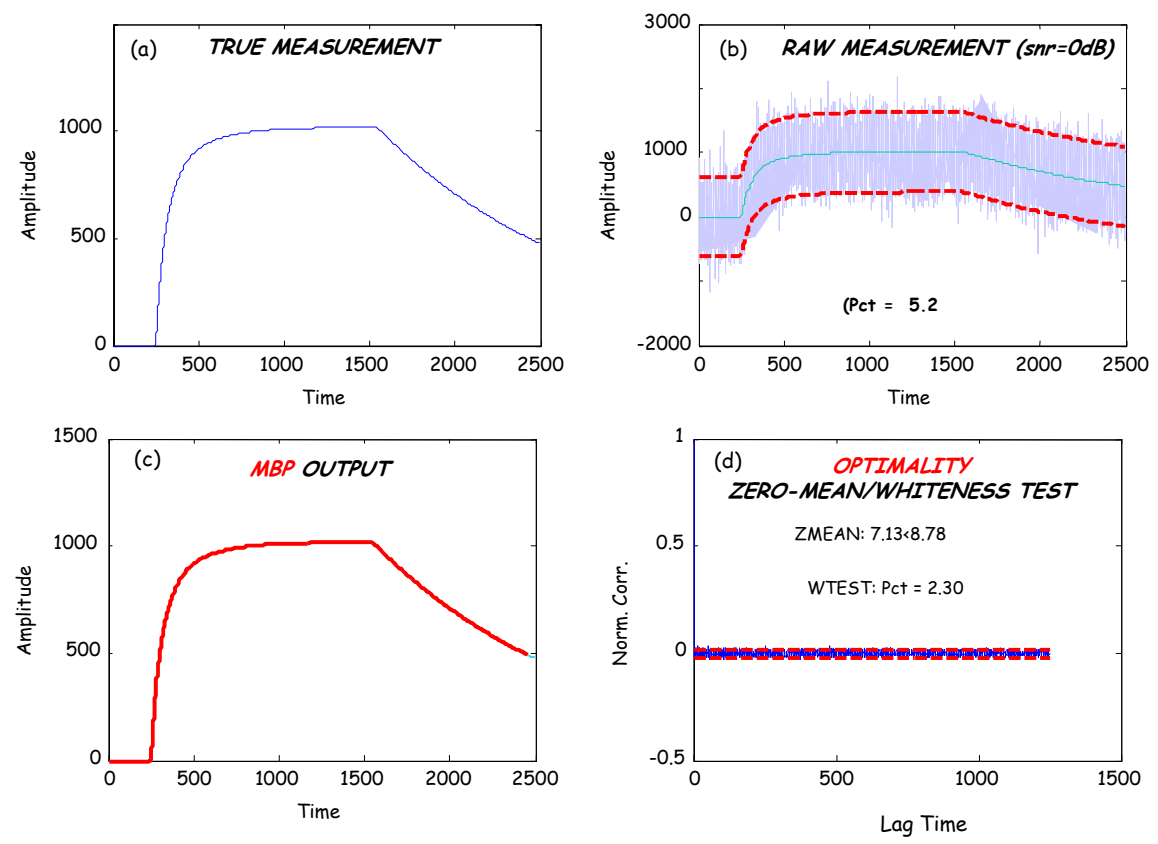

Fig. 38: MBP Design for Simulated Average Cantilever Data (0 dB SNR): (a) True measurement. (b) Simulated Gauss-Markov deflection measurement with bounds. (c) Enhanced deflection (MBP output). (d) Optimality tests (zero-mean/whiteness) results.
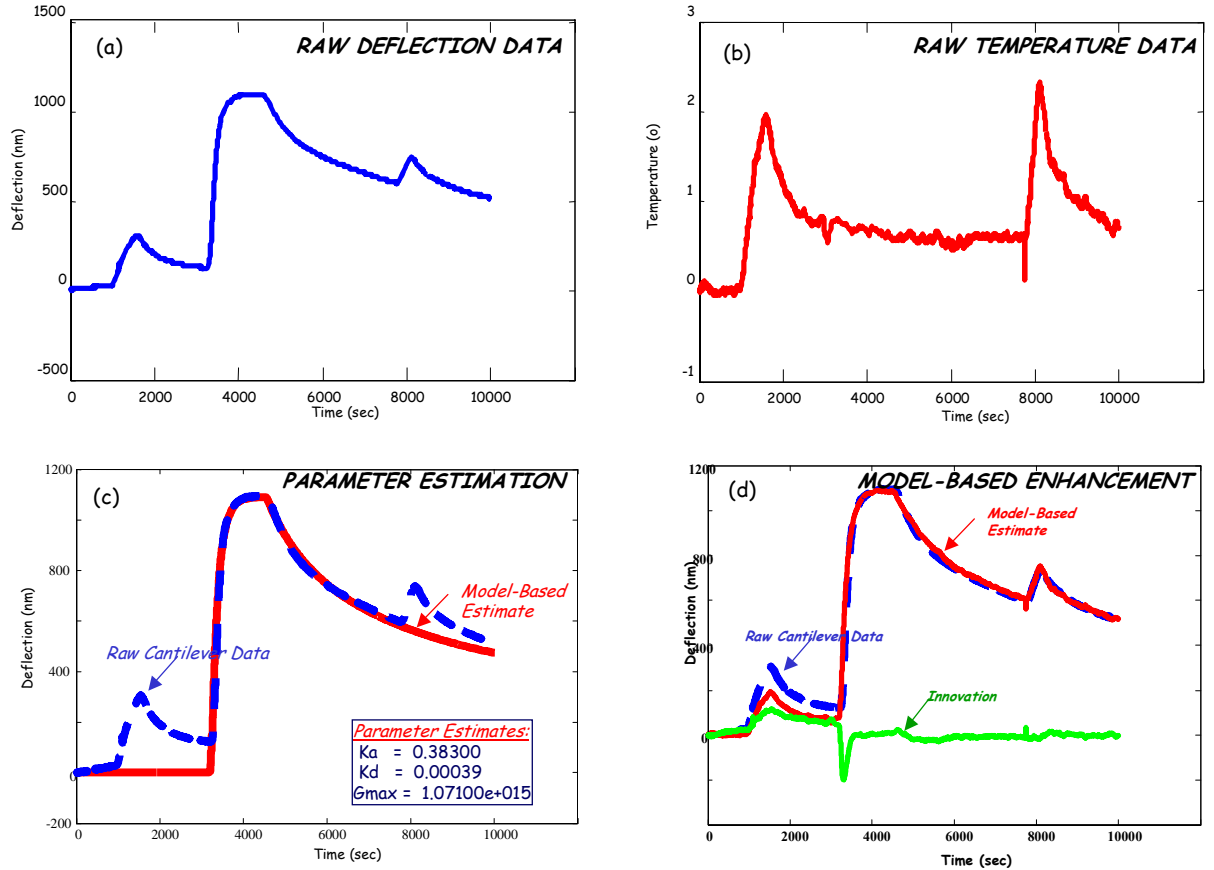

Fig. 39: Average Cantilever MBP Application to Measured Deflection Data. (a) Raw deflection data. (b) Raw temperature profile data. (c) Model-based parameter estimation fit and parameters. (d) Model-based enhancement including temperature. 
Next we developed the MBP for the multi-channel case using the same modelbased approach: simulation and application. We used the average model parameters developed over the entire cantilever array data set using the nonlinear least-squares model-based parameter estimator and applied it to the raw cantilever data to investigate its performance. We again used the MBP with the free energy as our piecewise constant parameter (state) and the nonlinear cantilever array model with 6 elements. We used the smoothed estimate of the temperature profile in our estimator as in the synthesized data case. We also used the estimated moduli in the Stoney equations as well as the average parameter estimates as before. The results are shown in Fig. 40 where we see the raw measured cantilever data, MBP estimates and the corresponding errors or innovations. Since the innovations are not zero-mean and white, the processor is not optimal; however, the results are quite reasonable except for the systematic bias error (constant) in the estimate. The dynamics appear to be captured by the model especially in cantilever 5 . The offset can be adjusted by selecting various combinations of elements in the $R_{v v}$ measurement noise covariance matrix, but this may be better suited to an adaptive implementation of the processor which will be pursued in future work. From the figure we note that the dynamics of the individual levers (on-set and off-sets) are quite close to the expected. This concludes the application to the data.
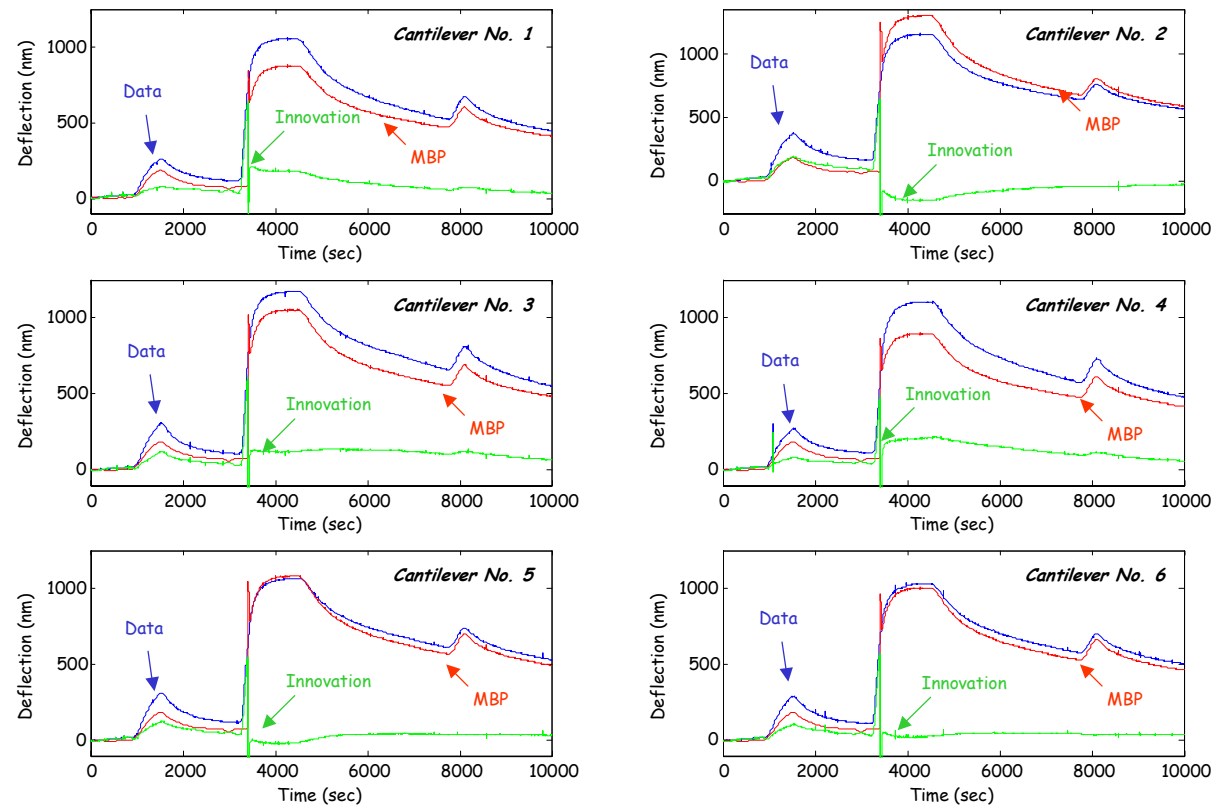

Fig. 40: MBP of Experimental Cantilever Array Data: Raw, Enhanced (MBP) and Residual (Innovation) results for each lever. 


\section{Summary}

We have developed a successful model-based approach to the micro-cantilever array signal enhancement problem. Experimental data were obtained from a multicantilever detection system, then quantitatively analyzed with mathematical tools from physical chemistry and solid state physics. We incorporated the results of this analysis directly into the generic signal processing approach. A proof-of-concept solution was created to parameterize our theoretical model, enabling us to test an average model. This model was then used to develop the MBP for enhancing noisy cantilever measurements. We investigated both data averaged over the array and the multi-channel cases.

Through simulations with additive Gaussian noise at SNR of $0,-20$, and $-40 \mathrm{~dB}$, we demonstrated the ability of the processor to extract the cantilever deflection response with a large improvement in signal gain $(\sim 80 \mathrm{~dB})$. We compared the processor to that of an "averager" (low-pass filter) comparing their performance. At each of these SNR the MBP demonstrated superior performance with an overall average processing gain of 40-60 dB over the averager. Finally, we applied the MBP to noisy, smoothed (averaged) cantilever data and demonstrated that the processor could perform quite well except for a bias error, which is easily corrected.

To demonstrate the full utility of the MBP for chemical sensing of low levels of signature chemicals associated with nuclear processing, necessary next steps are (1) verify the physical models used in this study for a variety of solvents and target molecules (this data has already been obtained as part of this study) (2) make use of control levers, and (3) extend the experimental library to include low concentrations of chemical targets of practical interest for sensing nuclear processes.

\section{ACKNOWLEDGEMENTS}

The authors gratefully acknowledge the contributions of LLNL scientists Brad Hart, Brandon Weeks, and Steve Velsko, who provided guidance on chemical sensing and cantilever experimental protocols, and of DHS Fellow Asher Sinensky, who performed important measurements and analysis for this project. Funding came from the NA-22 Exploratory Research Program managed by David Dye. This work was performed under the auspices of the Department of Energy by the University of California/Lawrence Livermore National Laboratory under contract W-7405-ENG-48. 


\section{Appendix A: Derivation of adsorption and desorption kinetics}

In this appendix, a different set of variables is used than was used in previous sections of the report. These parameters are more traditional in the field of kinetics, and so may be more familiar to some readers. In order to compare equations in this appendix with equations elsewhere in the report, use the following equivalencies:

$\mathrm{c}_{\mathrm{a}}=\Gamma_{\max }$

$\Theta=\Gamma / \Gamma_{\max }$

Consider a surface having a finite number of adsorption sites immersed in a gas or solution of potential sorbants. At equilibrium, the rate of sorption and desorption are equal:

$$
\mathrm{k}_{\mathrm{a}} \mathrm{c}_{0}\left(1-\Theta_{\mathrm{e}}\right)=\mathrm{k}_{\mathrm{d}} \mathrm{c}_{\mathrm{a}} \Theta_{\mathrm{e}}
$$

where $\mathrm{k}_{\mathrm{f}}$ is the forward rate constant (adsorption), $\mathrm{c}_{0}$ is the solution concentration, $\Theta_{\mathrm{e}}$ is the fraction of total adsorption sites occupied at equilibrium for this particular concentration, $\mathrm{k}_{\mathrm{r}}$ is the reverse rate constant (desorption), and $\mathrm{c}_{\mathrm{a}}$ is the total areal concentration of adsorption sites.

Solving for $\Theta$, we obtain the form of the traditional Langmuir isotherm:

$$
\Theta_{\mathrm{e}}=\mathrm{b} \mathrm{c}_{0} /\left(1+\mathrm{b} \mathrm{c}_{0}\right)
$$

where

$$
\mathrm{b}=\mathrm{k}_{\mathrm{a}} / \mathrm{k}_{\mathrm{d}} \mathrm{c}_{\mathrm{a}}
$$

Ordinarily, $\mathrm{b}$ is defined as $\mathrm{b}_{0} \exp (-\Delta \mathrm{H} / \mathrm{RT})$, where $\Delta \mathrm{H}$ is the enthalpy of adsorption. Using the standard Arrhenius formalism for the k's, we find

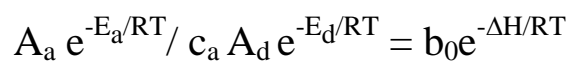

At which point it is obvious that $b_{0}=A_{f} / c_{a} A_{r}$ and $\Delta H=E_{a}-E_{d}$. The Langmuir isotherm has the property that equilibrium fractional surface coverage is linear with concentration at low concentration and reaches an asymptote of one at high concentration. Also note that the fractional coverage as a function of concentration is a function of temperature.

Now consider the rate of change in surface coverage away from equilibrium. The net change in fractional surface coverage is the difference between the forward and reverse rates:

$$
\mathrm{d} \Theta / \mathrm{dt}=\mathrm{k}_{\mathrm{a}} \mathrm{c}_{0}(1-\Theta)-\mathrm{k}_{\mathrm{d}} \mathrm{c}_{\mathrm{a}} \Theta
$$


Note that the net rate approaches zero as the surface concentration approaches its equilibrium value, when the forward and reverse rates by definition must become equal. This implies that one can neglect desorption when deriving $k_{a}$ only for very low surface coverages. One can factor the equation to get a relationship describing the rate as a function of the distance from equilibrium:

$$
\mathrm{d} \Theta / \mathrm{dt}=\mathrm{k}_{\mathrm{a}} \mathrm{c}_{0}\left(1-\Theta-\Theta / \mathrm{bc}_{0}\right)=\mathrm{k}_{\mathrm{a}} \mathrm{c}_{0}\left(1-\Theta\left(1+1 / \mathrm{bc}_{\mathrm{s}}\right)\right)=\mathrm{k}_{\mathrm{a}} \mathrm{c}_{0}\left(1-\Theta\left(\mathrm{bc}_{0}+1\right) / \mathrm{bc}_{0}\right)
$$

Comparing to Eq. A2, we find

$$
\mathrm{d} \Theta / \mathrm{dt}=\mathrm{k}_{\mathrm{a}} \mathrm{c}_{0}\left(1-\Theta / \Theta_{\mathrm{e}}\right)
$$

The rate goes to zero when $\Theta=\Theta_{\mathrm{e}}$. Although Eq. A7 is factored to approach equilibrium from the adsorption side, one can also factor Eq. A5 to approach equilibrium from the desorption side:

$$
\mathrm{d} \Theta / \mathrm{dt}=\mathrm{k}_{\mathrm{d}} \mathrm{c}_{\mathrm{a}}\left(-\Theta+(1-\Theta) b \mathrm{c}_{0}\right)
$$

If $\mathrm{c}_{0}=0$, one gets the trivial result that

$$
\mathrm{d} \Theta / \mathrm{dt}=-\mathrm{k}_{\mathrm{d}} \mathrm{c}_{\mathrm{a}} \Theta
$$

Note that all adsorption terms have fallen out automatically. Now consider the case where the concentration has been reduced (but not eliminated) so that the surface coverage is above its equilibrium value. The resulting approach to the new equilibrium value is given by

$$
\mathrm{d} \Theta / \mathrm{dt}=\mathrm{k}_{\mathrm{d}} \mathrm{c}_{\mathrm{a}} \mathrm{bc}_{0}\left(1-\Theta\left(1+\mathrm{bc}_{0}\right) / \mathrm{bc}_{0}\right)=\mathrm{k}_{\mathrm{d}} \mathrm{c}_{\mathrm{a}} \mathrm{bc}_{0}\left(1-\Theta / \Theta_{\mathrm{e}}\right)
$$

Note that $d \Theta / d t$ is negative in this form because $\Theta / \Theta_{e}$ is greater than one. One can eliminate $\mathrm{bc}_{0}$ in favor of $\Theta_{\mathrm{e}}$ by first inverting Eq. A2 to give

$$
\mathrm{bc}_{0}=\Theta_{\mathrm{e}} /\left(1-\Theta_{\mathrm{e}}\right)
$$

and then substituting Eq. A11 into the next to last equality, giving

$$
\mathrm{d} \Theta / \mathrm{dt}=\mathrm{k}_{\mathrm{d}} \mathrm{c}_{\mathrm{a}}\left(1-\Theta / \Theta_{\mathrm{e}}\right) \Theta_{\mathrm{e}} /\left(1-\Theta_{\mathrm{e}}\right)=-\mathrm{k}_{\mathrm{d}} \mathrm{c}_{\mathrm{a}}\left(\Theta-\Theta_{\mathrm{e}}\right) /\left(1-\Theta_{\mathrm{e}}\right)
$$

One observes an effective first-order desorption rate constant equal to the zeroconcentration value divided by the factor $\left(1-\Theta_{\mathrm{e}}\right)$.

Now consider the case where the apparent desorption order is not unity, which we observed for our gold-thiol system. This could be due to a difference in absorption energies for different sites, which would cause the activation energy and desorption rate constants to be different (Figure A1). 
We have shown previously [16] that a distribution of activation energies yields a deceleratory reaction that can be fitted well with an nth-order reaction having $\mathrm{n}>1$.

Formally, an nth-order reaction corresponds to a gamma distribution of frequency factors, but distributions of frequency factors and activation energies are difficult to distinguish over narrow temperature ranges. An important feature of the model in Figure A1 is that the non-unity reaction order would be observed only in the desorption direction, because the adsorption direction is characterized by a single activation energy.

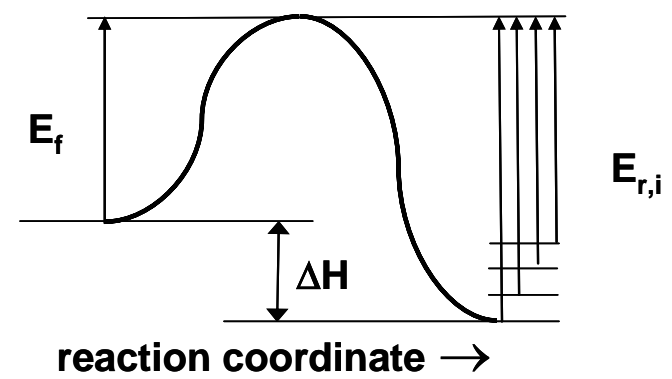

Figure A1. Conceptual model giving a distribution of reaction constants for desorption, which is reflected in an effective reaction order greater than one. 


\section{REFERENCES:}

1. Hagan, M.F., A. Majumdar, and A.K. Chakraborty, Nanomechanical forces generated by surface grafted DNA. Journal of Physical Chemistry B, 2002. 106(39): p. 10163-10173.

2. Halperin, A., A. Buhot, and E.B. Zhulina, Sensitivity, specificity, and the hybridization kinetics of DNA chips. Biophysical Journal, 2004. 86(2): p. 718730.

3. Lavrik, N.V., et al., Gold Nano-structures for transduction of biomolecular interactions into micometer scale movements. Biomedical Microdevices, 2001. 3(1): p. 35-44.

4. Marie, R., et al., Adsorption kinetics and mechanical properties of thiol-modified DNA-oligos on gold investigated by microcantilever sensors. Ultramicroscopy, 2002. 91(1-4): p. 29-36.

5. Georgiadis R., Peterlinz K.P., and Peterson A.W. Quantitative Measurements and Modeling of Kinetics in Nucleic Acid Monolayer Films using SRP Spectroscopy. J. Am. Chem. Soc. 2000. 122, 3166-3173.

6. Karpovich, D.S. and G.J. Blanchard, Direct measurement of the adsorption kinetics of Alkanthiolate self-assembled monolayers on mirocrystalline gold surfaces. Langmuir, 1994. 10(10): p. 3315-3322.

7. $\quad$ Roark and Young, Formulas for Stress and Strain. p. Sect. 7.2.

8. Sader, J.E., Surface stress induced deflections of cantilever plates with applications to the atomic force microscope: Rectangular plates. Journal of Applied Physics, 2001. 89(5): p. 2911-2921.

9. Haiss, W., Surface stress of clean and adsorbate-covered solids. Reports on Progress in Physics, 2001. 64(5): p. 591-648.

10. Candy, J., Signal Processing: The Model-Based Approach. 1986, New York: McGraw-Hill.

11. Trees, H.V., Detection, Estimation and Modulation Theory. Vol. 1, New York: Wiley.

12. Gill, P., W. Murray, and M. Wright, Practical Optimization. 1981, New York: Academic Press.

13. Peterlinz, K.A. and R. Georgiadis, In situ kinetics of self assembly by surface plasmon resonance spectroscopy. Langmuir, 1996. 12: p. 4731-4740.

14. McKendry, R., et al., Multiple label-free biodetection and quantitative DNAbinding assays on a nanomechanical cantilever array. Proceedings of the National Academy of Sciences of the United States of America, 2002. 99(15): p. 9783-9788.

15. Candy, J. and P. Candy, SSPACK_PC, a model-based signal processing package on personal computers. 1993, DSP Applications.

16. Burnham, A.K. and R.L. Braun, Global kinetic analysis of complex materials. Energy and Fuels, 1999. 13(1): p. 1-22. 